\title{
Endovascular repair for thoracoabdominal aortic aneurysms: current status and future challenges
}

\author{
Emanuel R. Tenorio, Marina F. Dias-Neto, Guilherme Baumgardt Barbosa Lima, Anthony L. Estrera, \\ Gustavo S. Oderich
}

Department of Cardiothoracic \& Vascular Surgery, Advanced Aortic Research Program, McGovern Medical School at The University of Texas Health Science Center at Houston (UTHealth), Houston, TX, USA

Correspondence to: Gustavo S. Oderich, MD. Professor and Chief of Vascular and Endovascular Surgery, Department of Cardiothoracic and Vascular, McGovern Medical School at UTHealth, 6400 Fannin, Suite \#2850, Houston, TX 77030, USA. Email: gustavo.oderich@uth.tmc.edu.

\begin{abstract}
Open surgical repair has been the gold standard for treatment of thoracoabdominal aortic aneurysms (TAAA). Currently, open surgical repair has been reserved mostly for young and fit patients with connective tissue disorders, using separate branch vessel reconstructions instead of 'island' patches, and distal perfusion instead of a 'clamp and go' technique. Endovascular repair has gained widespread acceptance because of its potential to significantly decrease morbidity and mortality. Several large aortic centers have developed dedicated clinical programs to advance techniques of fenestrated-branched endovascular aortic repair (FB-EVAR) using patient-specific and off-the-shelf devices, which offers a less-invasive alternative to open repair. Although FB-EVAR was initially considered an option for older and frail patients, many centers have expanded its indications to any patient with suitable anatomy and no evidence of connective tissue disorders, independent of their clinical risk. In this article, we review current techniques and outcomes of endovascular TAAA repair.

Keywords: Thoracoabdominal aortic aneurysms (TAAA); open surgical repair; fenestrated-branched endovascular repair; fenestrated-branched endovascular aortic repair (FB-EVAR)
\end{abstract}

Submitted Sep 07, 2021. Accepted for publication Oct 26, 2021.

doi: 10.21037/acs-2021-taes-24

View this article at: https://dx.doi.org/10.21037/acs-2021-taes-24

\section{Introduction}

Thoracoabdominal aortic aneurysms (TAAA) account for approximately $10 \%$ of all aortic aneurysms, and present a formidable technical challenge associated with high morbidity and mortality (1). Although most aneurysms are degenerative, advances in molecular diagnosis have identified several genetically triggered aortic diseases associated with aortic aneurysms and dissections (2). Open surgical repair remained the gold standard for treatment of TAAAs for more than six decades. Techniques have been refined since the original contributions of $\mathrm{E}$. Stanley Crawford, which focused on organ protection and prevention of recurrent aneurysms (3). In the last three decades, techniques of distal organ protection, routine cerebrospinal fluid drainage and separate branch vessel reconstruction largely replaced the 'clamp and go' method with 'island patch' reconstruction (4). Despite these advances, a thirty-day mortality of $7-17 \%$ is still reported in the largest open surgical series, with significant morbidity including spinal cord injury (2-14\%), dialysis (2-32\%), tracheostomy (4-12\%) and early reoperations for postoperative complications (2-25\%) (5).

Endovascular TAAA repair was introduced in the late 1990s and early 2000s using reinforced fenestrations or directional branches, offering a less invasive approach to open surgical repair (6). The technique has evolved from using physician-modified endovascular grafts (PMEGs) to patient-specific and off-the-shelf devices. Along with several improvements in device design, several dedicated aortic centers have perfected technique and perioperative care with continued improvement in clinical outcomes (7). Although device access remains limited in the United 


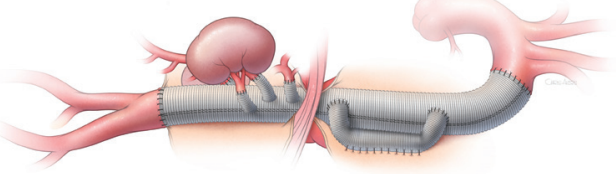

Figure 1 Open surgical repair of an Extent II thoracoabdominal aortic aneurysm with thoracolaparotomy incision, revascularization of the renal-mesenteric arteries using end-to-end anastomoses with loop graft for intercostal arteries reattachment. (Printed with permission from Chris Akers for the Annals of Cardiothoracic Surgery. All copyrights reserved for McGovern Medical School at UTHealth, Houston, Texas).

States, in Europe, United Kingdom, Canada, Asia and South America, FB-EVAR is commonly used as first line treatment for complex aortic aneurysms. The last European Clinical Practice Guidelines for management of aortoiliac aneurysms recommends FB-EVAR as the preferred option for suprarenal aortic aneurysms when feasible (8). The role of FB-EVAR on the treatment of TAAAs has not yet been addressed by any of the society guidelines but growing evidence demonstrates that results in large centers rival any of the open surgical series $(9,10)$.

\section{Evolution of FB-EVAR technique}

There are several potential advantages of an endovascular approach. These procedures can be performed percutaneously using the transfemoral approach, avoiding the need for thoracolaparotomy incision, division of the diaphragmatic muscle (Figure 1), left heart or cardiopulmonary bypass and aortic cross clamping. There is uninterrupted aortic flow and perfusion of the renal, mesenteric and lower extremity vessels. Therefore, the procedure fundamentally reduces physiological demand, end-organ ischemia, blood loss, fluid requirements and transfusion of blood products. Nonetheless, despite these advances, there are shortcomings imposed by the technique and other anatomical constraints. Small vessels, such as intercostal arteries, cannot be reconstructed by endovascular means with rare exceptions, raising concern that extensive aortic coverage may increase the risk of spinal cord injury. Furthermore, catheter and wire manipulations in the midst of atheromatous debris may result in embolization and endorgan damage.

Fenestrated and branched stent grafts were first applied to patients with short infrarenal necks using devices with one or two non-reinforced fenestrations. The fenestrations were not aligned by bridging stents, which led to loss of target vessels due to fabric shuttering. Subsequently, modifications of the technique included reinforcement of fenestrations, diameter reducing ties, a separate distal bifurcated device from the proximal fenestrated component and use of balloon-expandable covered stents for alignment of fenestrations. Low-profile devices and preloaded systems have been added in the last decade. Currently, industrymanufactured designs can be categorized as patient-specific devices and off-the-shelf stent grafts (Figure 2).

Preloaded wires and catheters are intended to provide direct access to branches and fenestrations, thereby decreasing the need to manipulate catheters and potentially shortening procedure time. These systems have been widely used with fenestrated devices, such as the p-Branch ${ }^{\circledR}$ and other patient-specific Zenith ${ }^{\circledR}$ fenestrated stent grafts (Cook Medical, Bloomington, IN) and, more recently, have been modified to allow the options of femoral and/ or brachial access for TAAAs. The low-profile device with upper extremity preloaded guidewire system (LP-PGS) uses one or two preloaded 0.018 -inch wires that are accessible via femoral and brachial approaches (thoracoabdominal preloaded low profile, TPLP) or brachial only approach (thoracoabdominal preloaded system, TPDS). The device utilizes a $20 \mathrm{Fr}$ delivery system with a long $8 \mathrm{Fr}$ nosecone that is connected to the tip of the $20 \mathrm{Fr}$ cannula. Alternatively, the device may be loaded into an 18 Fr delivery system with one or two preloaded wires that exit via the top of the delivery system, akin to what has been described with PMEGs (11). Finally, the Gore TAMBE ${ }^{\circledR}$ TAAA device has four preloaded stylets that allow advancement of 0.014-18inch wires to each directional branch. Independent of which design is used, brachial preloading requires through-andthrough brachial-femoral access and a $12 \mathrm{Fr}$ brachial sheath (Figure 3) (12).

The largest worldwide FB-EVAR experience has been with patient-specific devices using the Cook Zenith ${ }^{\circledR}$ platform (Cook Medical Inc, Brisbane Australia and Denmark), with more than 55,000 implants (Personal communication, Cook Medical Inc.). The technique was pioneered in 1997 in Perth, Australia, by Tom Browne, Michael Lawrence Brown and David Hartley, with the first clinical implant in 1998 by John Anderson. In the last decade, several other manufacturers have developed their own iterations. At present, patient-specific devices include the Zenith Fenestrated platform (Cook Medical Inc, Bloomington, IN), the Fenestrated Anaconda (Vascutek, 


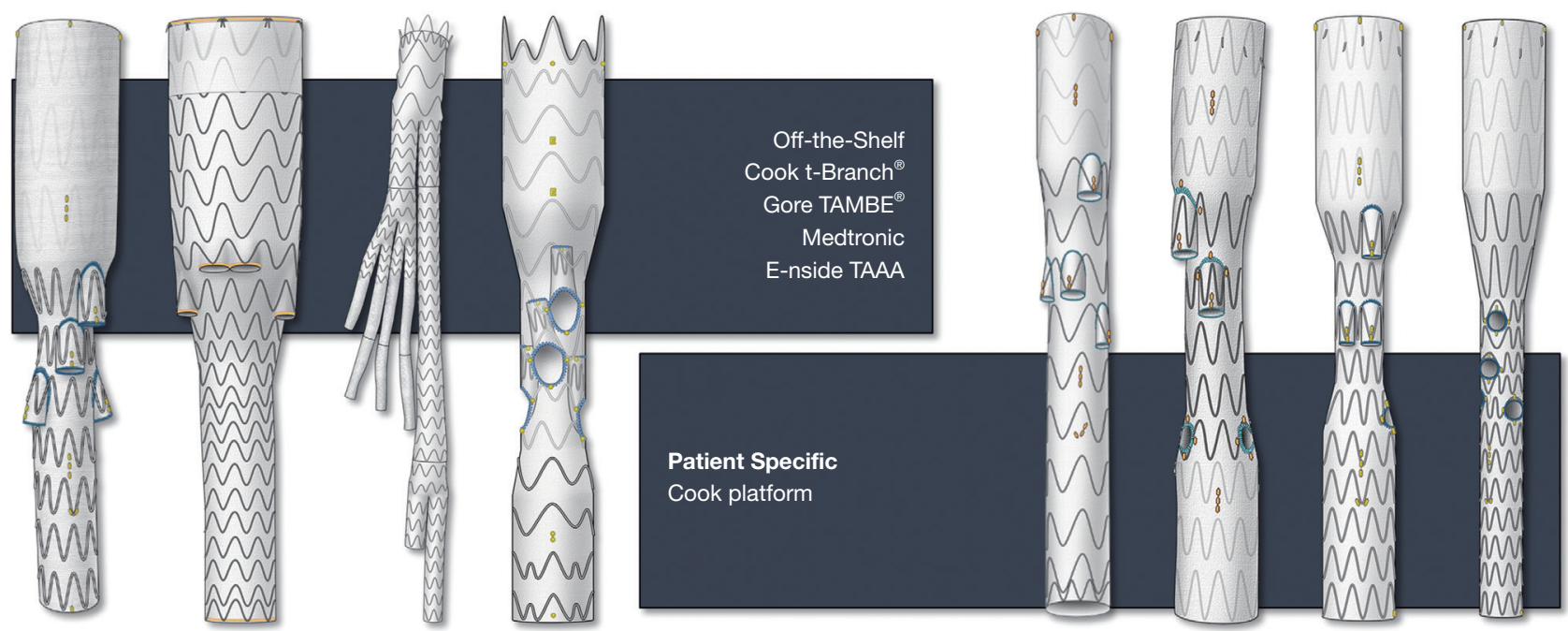

Figure 2 Illustrations of the current off-the-shelf devices and the patient-specific platform for endovascular thoracoabdominal aortic aneurysm repair.

A

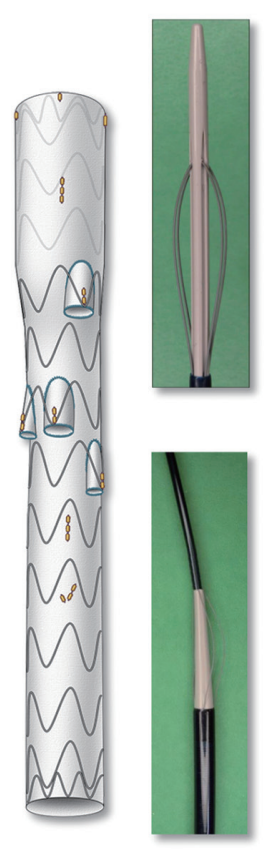

B

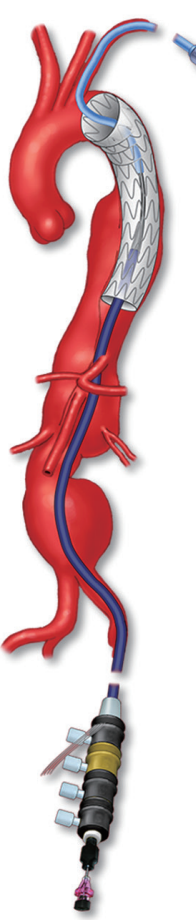

D

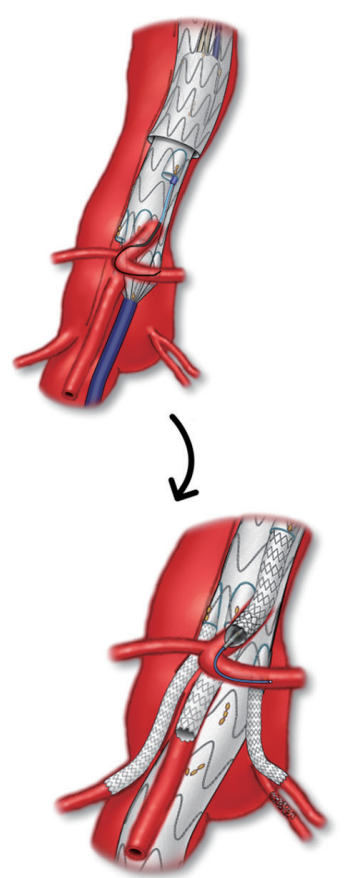

E

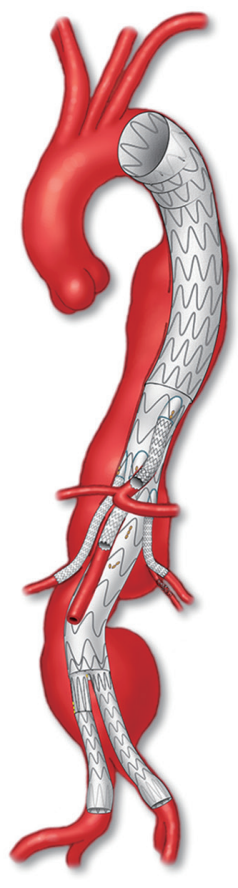

Figure 3 Patient-specific branched stent graft with thoracoabdominal preloaded delivery system (TPDS). (A) The preloaded wires are housed in the distal nose cone; (B) the device is introduced via the femoral approach using a brachial-femoral wire and the long nosecone exits via the brachial sheath; (C) once the sheath is removed, the two loops of preloaded 0.014-inch guidewires are revealed and cut to form four wires, which are labeled to its intended target vessel; (D) the wires are used for sequential target catheterization and stenting using staggered technique; (E) the repair is completed with placement of distal bifurcated device and iliac extensions. 
A

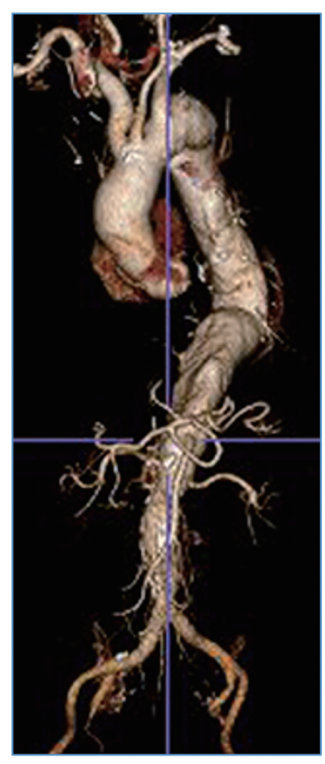

B

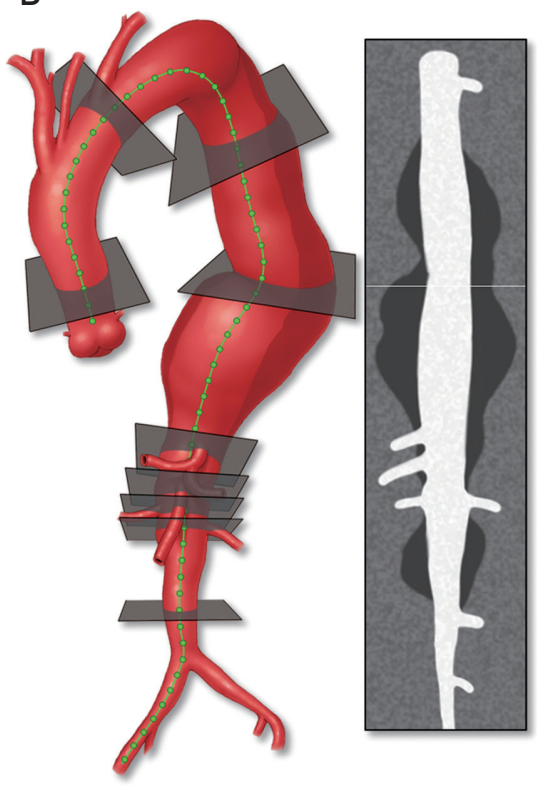

C

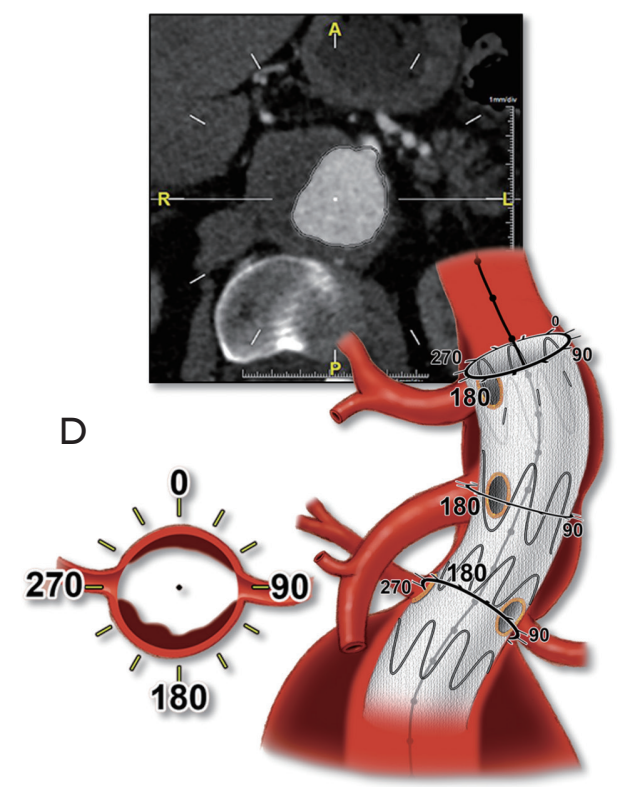

Figure 4 Three-dimensional (3D) computed tomography angiography (A) is analyzed using centerline of flow measurements (B) for measurements of lengths while orthogonal views are used for analysis of vessel origin in axial plane (C). Precise planning of complex endovascular repair is done using the triangulation technique (D).

Terumo Aortic, Inchinnan, Scotland) and Jotec Cryolife TAAA device (Jotec GmbH, Hechingen, Germany), whereas off-the-shelf devices include the Zenith ${ }^{\circledR}$ t-Branch ${ }^{\circledR}$ TAAA stent graft (Cook Medical, Bloomington, IN, USA), GORE EXCLUDER thoracoabdominal branch endoprosthesis (TAMBE, W.L. Gore \& Associates Inc, Flagstaff, AZ) (13), Medtronic Valiant modular branched graft (Medtronic, Minneapolis, MN), Colt device and E-nside TAAA Multibranch Stent Graft System (Jotec GmbH, Hechingen, Germany).

\section{Planning and technical assessment}

Preoperative planning is the foundation for any successful endovascular aortic repair. Detailed planning requires triangulation of the renal and mesenteric vessels using the centerline technique in a three-dimensional workstation (Figure 4). Independent of the stent design, the following measurements are obtained:

* Assessment of proximal and distal sealing zones (size, surface, angulation);

* The longitudinal distances between target vessels, radial or clock positions;

* Inner aortic luminal diameter at each target vessel origin for calculation of arc lengths and measurement of fenestration gap distance, defined by the distance from a fully deployed endograft to the target vessel origin;

* Target vessel patency, distance to first branch bifurcation, diameter, angulation and trajectory;

* Patency of spinal collateral networks, including intercostal, subclavian, vertebral and hypogastric arteries;

- Evaluation of access vessels, including common femoral, iliac and brachial arteries; and

* Evaluation of aortic atherosclerotic burden and vessel wall calcification.

Although clinicians may be tempted to delegate planning to the manufacturer, this is a risky strategy at best, and does not allow proper development of technical strategies. Although accurate measurements will be provided, the plan will be devoid of clinical judgment and compromise. Such grafts may be a 'true fit' but prove impossible to implant. Nevertheless, consultative engagement with the planning center, rather than delegation provides invaluable insight. Later, these centers can be used as an expert resource for planning more complex repairs. When used irresponsibly, planning centers can become a way for inexperienced 
physicians outside of high-volume centers to gain access to a technique that they may never fully master.

There has also been continuous improvement in the development of advanced imaging support to perform complex endovascular aortic procedures. The initial cases were performed with portable $\mathrm{C}$-arms, and sizing was achieved using simple axial imaging. Currently, threedimensional workstations and high-quality operative imaging in dedicated hybrid operative rooms with flat panel detectors and fusion imaging software are recommended for these procedures.

The intraoperative management of advanced imaging equipment requires specific training for the operators (surgeons, radiologists and radiographers). Interventionists require detailed knowledge of the fluoroscopic options, including imaging settings, angulation, radiation exposure and protection. Modern hybrid operating rooms with capability of onlay fusion and high-definition cone beam computed tomography (CBCT) have been instrumental in optimizing the imaging needed for accurate deployment of the aortic stent graft and its side branches, while minimizing radiation exposure and allowing intraoperative technical assessment. These techniques have been shown to decrease radiation exposure and the operator's effective dose during FB-EVAR (14). In addition, CBCT allows immediate on-table assessment to identify technical problems requiring revision before the patient leaves the operating room, thereby avoiding unnecessary secondary interventions. Traditional 2D completion digital subtraction angiography (DSA) alone provides inadequate assessment of the technical result after FB-EVAR. A complete contrastenhanced CBCT with rotational DSA detects $93 \%$ of technical problems and eliminates the need for pre-dismissal CTA (15). Currently, we only obtain a pre-dismissal CTA if clinically indicated.

\section{Patient-specific devices}

Patient-specific TAAA devices are tailored to address variability of aortic and visceral branch anatomy $(16,17)$. The Cook Zenith Fenestrated (Cook Medical Inc, Bloomington, IN) stent graft became available for commercial use in Europe in 2005. In the US, the Food and Drug Administration (FDA) approved the Zenith Fenestrated device for treatment of patients with shortneck abdominal aortic aneurysms (AAA) in 2012. Contrary to the version used by the rest of the world, which has no limitation on the number of fenestrations or directional branches, the US-version of the Cook Zenith Fenestrated device has major anatomical constraints. It is only approved for up to three fenestrations, of which two can be of the same type. In addition, scallops are single-wide $(10 \mathrm{~mm})$ and large fenestrations are not strut free or reinforced by nitinol rings. In the US, there are ten centers with ongoing physician-sponsored investigational device exemption (PS-IDE) protocols that allow access to the entire pipeline of patient-specific and off-the-shelf Cook fenestrated and branched endografts. These devices can be manufactured with any number and combination of fenestrations or directional branches. Fenestrations include double-wide scallops $(20 \mathrm{~mm})$, large $(8 \mathrm{~mm} \times 8 \mathrm{~mm})$ or small $(6 \mathrm{~mm}$ $\times 6 \mathrm{~mm})$. Directional branches can be down-going, upgoing, external or internal. Other adjuncts include diameter tapering to adjust for variations in luminal diameter, diameter reducing sutures, preloaded systems and inverted limb bifurcated devices. A limitation of patient-specific devices is the time delay needed for manufacturing the device, which averages six to eight weeks.

\section{Off-the-shelf devices}

Off-the-shelf, multi-branched TAAA devices uniformly apply the concept of four down-going directional branches to incorporate the celiac axis, superior mesenteric artery (SMA) and both renal arteries. The Cook Zenith ${ }^{\circledR}$ t-Branch ${ }^{\circledR}$ stent graft (Cook Medical Inc., Bjaeverskov, Denmark) is a $202 \mathrm{~mm}$ length device with proximal diameter of $34 \mathrm{~mm}$ and distal diameter of $18 \mathrm{~mm}$ (Figure 5) (17). The celiac and SMA directional branches are $8 \mathrm{~mm}$ in diameter and located 99 and $117 \mathrm{~mm}$ distal to the top edge at one and twelve o'clock positions, respectively. The right and left renal artery cuffs are $6 \mathrm{~mm}$ in diameter and located $135 \mathrm{~mm}$ distal to the top edge at ten and three o'clock positions, respectively. The device has a $22 \mathrm{Fr}$ delivery system and no preloaded wires or catheters $(18,19)$.

The GORE EXCLUDER Thoracoabdominal Branch Endoprosthesis (TAMBE; W. L. Gore \& Associates, Flagstaff, Arizona) is an off-the-shelf, modular system composed of a proximal, multibranch stent graft, a distal bifurcated component and iliac limb extensions. It has four antegrade portals that can be accessed through the brachial approach. The proximal device has a diameter of 31 or $37 \mathrm{~mm}$, length of $160 \mathrm{~mm}$ and distal diameter of $20 \mathrm{~mm}$ (Figure 6). The device has four pre-loaded stylets intended for advancement of 0.014-18-inch guidewires, which are then loaded into a tri-lumen transbrachial catheter for access 
A

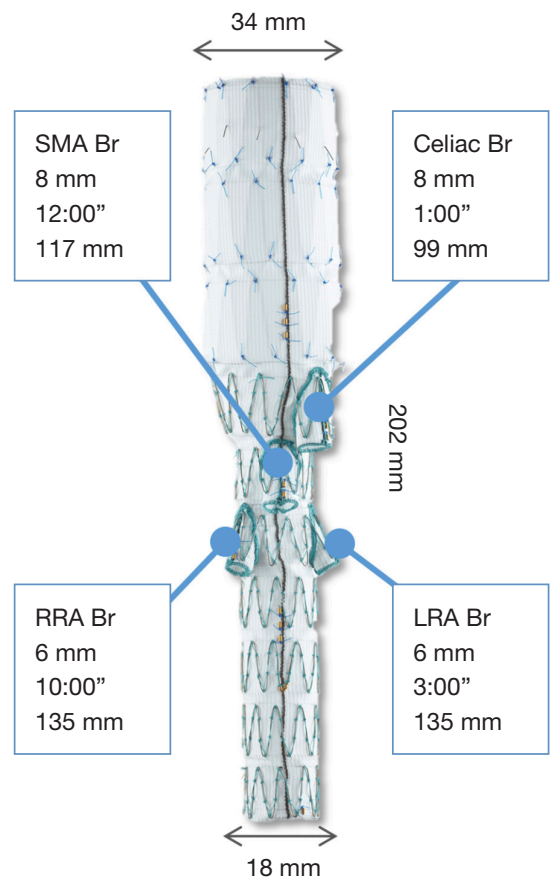

B

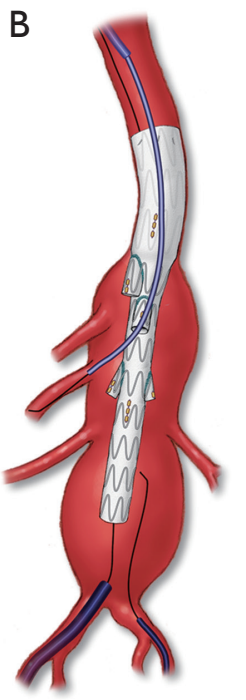

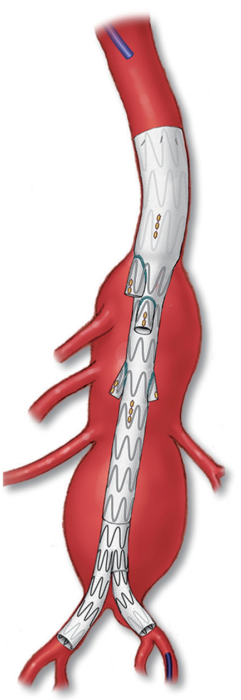
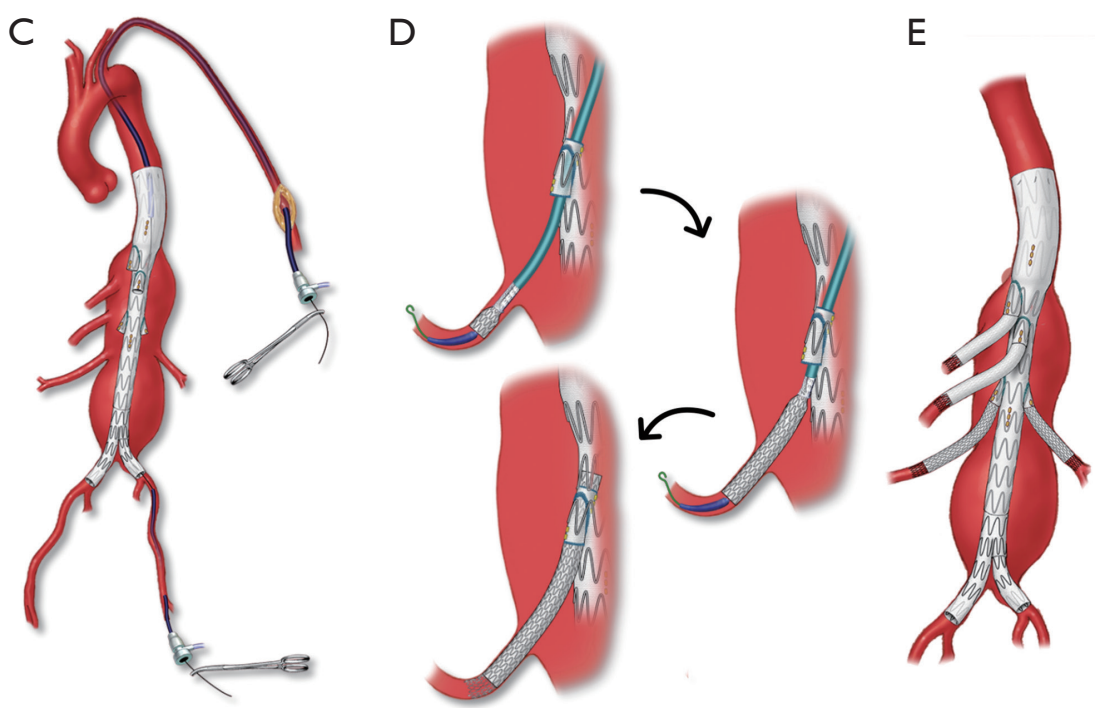

Figure 5 The multi-branch device is designed with four down-going directional branches intended for incorporation of the celiac artery (CA), superior mesenteric artery (SMA) and both renal arteries (A). The stent graft is introduced via the femoral approach and deployed with the directional branches approximately $2 \mathrm{~cm}$ above the target vessel. The distal bifurcated device and iliac limb extensions are placed, and flow is restored to the lower extremities (B). A 12 Fr branchial sheath is advanced to the descending thoracic aorta (C). The renal directional branches are sequentially catheterized and stented using self-expandable stent grafts (D). The repair is completed with placement of the celiac and superior mesenteric artery stent grafts (E).

via the brachial approach. The device requires a $20 \mathrm{Fr}$ or $22 \mathrm{Fr}$ introducer sheath. The device is intended to be used in conjunction with the Gore Viabahn Balloon Expandable Endoprosthesis (VBX - W. L. Gore \& Associates, Flagstaff,
Arizona) as the bridging stent for renal and mesenteric vessels. The TAMBE device is currently under investigation in a pivotal pre-market approval (PMA) study that is seeking indication for use for complex abdominal and TAAAs (13). 
A

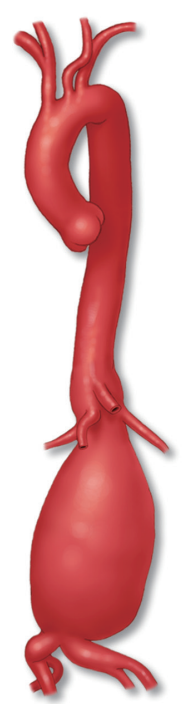

B

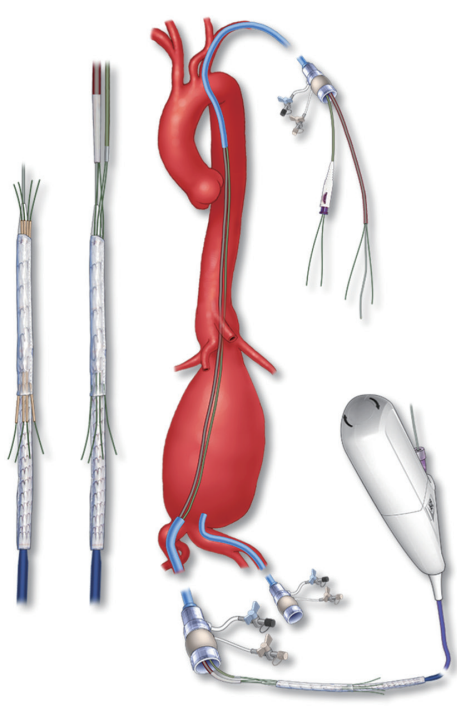

C

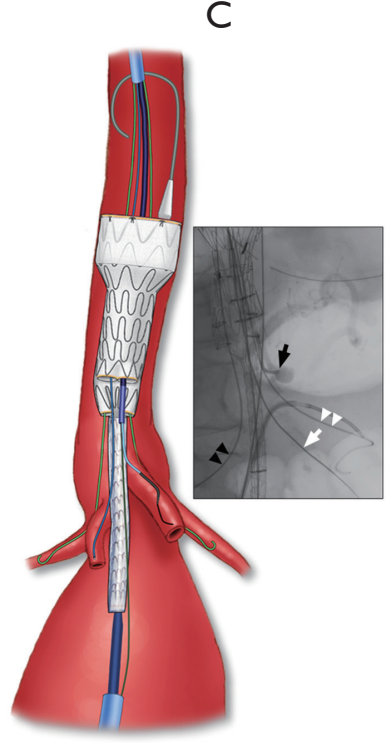

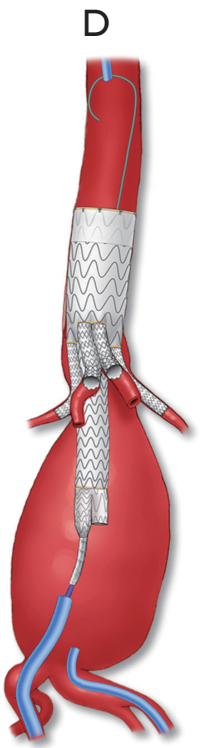

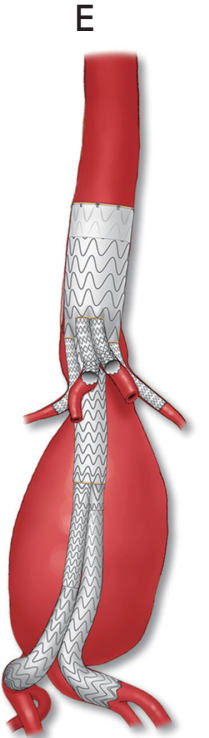

Figure 6 The TAMBE with four antegrade portals has four preloaded removable guidewire tubes (RGTs), which allowed advancement of 0.014- to 0.018-inch guidewires (A). Using the brachiofemoral guidewire, a GORE Tri-Lumen Catheter (Tri-Lumen; W. L. Gore \& Associates) and a "buddy" catheter were advanced from the brachial approach and exteriorized through the femoral sheath. The TAMBE is loaded into the main brachiofemoral guidewire, whereas separate 0.014- to 0.018-inch guidewires are sequentially introduced into the four preloaded RGTs and the Tri-Lumen and buddy catheters. It is critical to label each of the guidewires to the correct portal and target vessel (B). The device has a stepwise deployment system, which allowed the top part to be partially constrained and the distal portion of the device to be completely constrained to facilitate branch vessel catheterization. After limited angiography, the top portion of the aortic device is partially deployed and partially expanded toward the aortic wall. Each portal is sequentially catheterized using the preloaded guidewires. A 7 Fr Raabe hydrophilic sheath (Cook Medical) is sequentially advanced through the brachial or axillary approach into each of the antegrade portals. Once the sheath is distal to the portal, a 5 Fr buddy catheter is used to selectively catheterize the target vessel (arrows, C). The procedure is completed by placement of a distal bifurcated device, contralateral and ipsilateral iliac limb extensions, and balloon dilation of the landing zones and attachment sites $(\mathrm{D}, \mathrm{E})$.

\section{Prevention of spinal cord injury}

Paraplegia is one of the most devastating complications of TAAA repair. Although reimplantation of patent segmental arteries is possible during open surgical repair, these vessels are usually too small to allow placement of bridging stents. Spinal cord ischemia often results from multiple superimposed insults $(20,21)$. Some of the recognized factors include hemodynamic deterioration leading to spinal cord infarction secondary to loss of intercostal arteries coupled with insufficient collateral network; microembolization from catheter or device manipulations and reperfusion injury and spinal cord edema. Traditionally, embolization has been considered a relatively uncommon cause. However, most recently, microemboli have been increasingly recognized as a cause of spinal cord ischemia. Tanaka and associates reported that $80 \%$ of patients with spinal cord injury (SCI) following TAAA repair had scattered MRI lesions consistent with infarction from microembolization (22).

Recent experimental and clinical studies have shown that staged segmental arterial coverage of the aorta allows rapid recruitment of the spinal collateral networks (23). Bischoff and associates reported no spinal cord injury in pigs randomized to staged coverage compared to $50 \%$ rate of paraplegia in pigs treated in a single stage (24). Subsequently, the Cleveland Clinic group reported that internal iliac artery occlusion was an independent predictor of immediate spinal cord injury and lack of recovery, however staged thoracoabdominal aortic repair reduced early mortality and rates of paraplegia $(21,25)$. Other potential benefits of staged repair for thoracoabdominal aneurysms include earlier restoration of pelvic and lower extremity blood flow, lower rates of microembolization and changes 
A

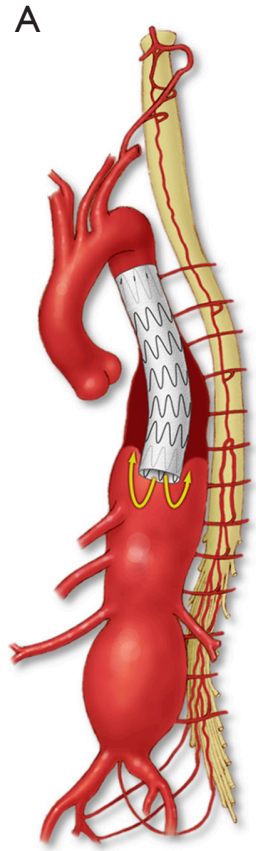

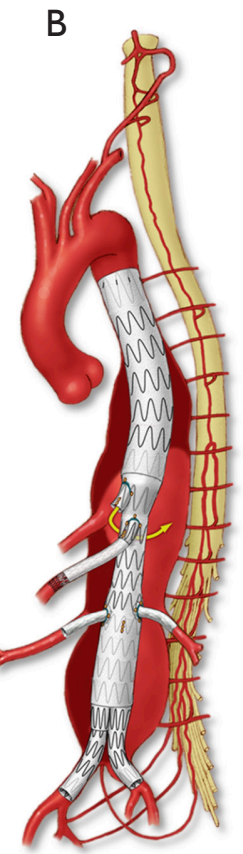
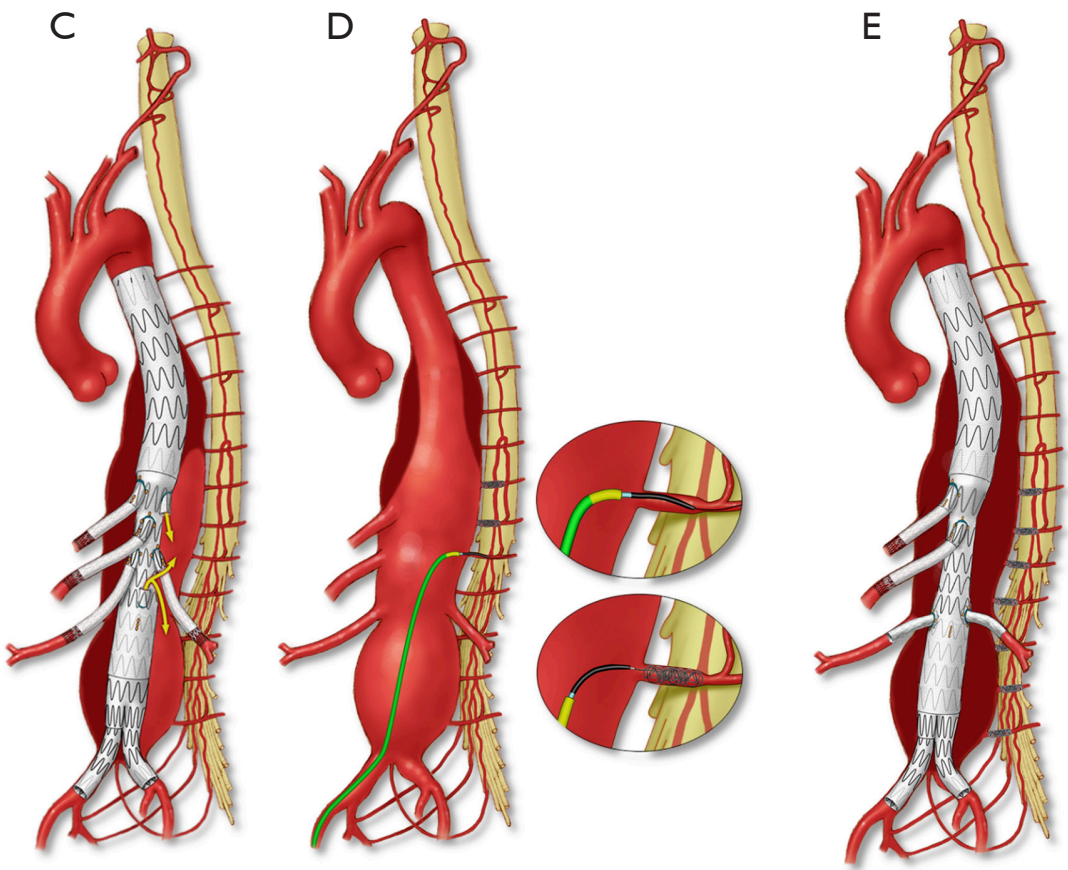

Figure 7 Staging strategies include proximal thoracic endovascular aortic repair from zone 3 to zone 5 (A), temporary aneurysm sac perfusion (TASP) via unstented directional branch (B) or pre-fashioned perfusion branches (C), and minimally invasive staged segmental artery coil embolization (MIS ${ }^{2} \mathrm{ACE}$ ) with sequential embolization of intercostal and lumbar arteries (D). Preconditioning of spine collateral network using one of these techniques is followed by definitive completion endovascular repair (E).

in systemic inflammatory response during the second-stage procedure. Although further clinical experience is needed to confirm the benefit of staging, current evidence suggests that a staged repair decreases mortality and SCI during endovascular TAAA repair $(23,25,26)$.

\section{Staging strategies}

Preconditioning of spinal cord collateral networks is the mainstay of staging strategies to reduce the risk of spinal cord ischemia. These techniques are recommended for more extensive aneurysms involving the entire thoracoabdominal aorta (Crawford Extent I and II TAAAs) and are used selectively for less extensive aneurysms. Staging can be performed using one of three strategies: selective catheter embolization of intercostal arteries; temporary aneurysm sac perfusion; or proximal thoracic endovascular aortic repair (Figure 7).

Minimally invasive segmental artery coil embolization (MIS $\left.{ }^{2} \mathrm{ACE}\right)$ is a term coined by Chris Etz to describe selective preventive catheter-guided embolization and occlusion of segmental arteries in order to promote the spinal collateral network. This technique has demonstrated promising results in a limited single-center experience (27) and is currently under investigation in a prospective multicenter randomized trial. Potential shortcomings are the need for multiple staging endovascular procedures with has risks of embolization and spinal ischemia, and the lack of reimbursement. Staged coverage of the thoracic aorta with TEVAR is an appealing alternative. This may be done using commercially approved thoracic stent grafts or specially designed devices with a distal flared segment, akin to 'bell bottom' flared iliac limbs. Often in these cases there is a distal type IB endoleak. However, most often, the proximal thoracic aneurysm sac thromboses, similar to aortic dissections treated by coverage of the proximal entry tear (28). Finally, temporary aneurysm sac perfusion (TASP) implies that an intentional endoleak is left upon completion of the index FB-EVAR procedure allowing perfusion of intercostal and lumbar arteries. This is achieved using perfusion branches that are integrated in the main custom-made aortic stent graft or, alternatively, one of the directional branches or the contralateral limb of the bifurcated stent graft. The perfusion branch is left patent in the initial procedure and is occluded a few days later using an Amplatzer ${ }^{\mathrm{TM}}$ plug, which can be deployed 
under local anesthesia. Limitations of TASP is the potential risks of increased sac pressure due to poor outflow via small segmental arteries and disseminated intravascular coagulopathy from a large endoleak.

Staging may also be needed in patients with extensive disease involving the aortic arch. In these cases, cervical debranching procedures-or a total arch reconstruction using the elephant trunk technique-may be needed to allow a suitable landing zone for distal endovascular repair (29). Our preference has been to use TEVAR as a first-stage strategy, allowing coverage of proximal intercostal arteries and minimizing the extent of the second stage procedure. The first step involves coverage of the descending thoracic aorta with a thoracic endovascular graft down to the level of the celiac axis. For patients with suitable aortic segments in zones 3 and 4 , the procedure is usually a straightforward stenting of the thoracic aorta with one or two endografts, tapering the diameter to $30-34 \mathrm{~mm}$ in preparation for the definitive repair. Distally, the thoracic aorta is covered just above the celiac artery, leaving a type IB endoleak prior to the second stage procedure. If the aorta is diseased up to the left subclavian artery, the landing zone may need to be extended more proximally into zone 2 . In these cases, left subclavian artery revascularization is routinely performed using either a left carotid-left subclavian bypass or a retrograde subclavian branch. If the patient is not an ideal candidate for total arch reconstruction, extension into zone 0 can also be performed using an endovascular approach with two or three inner branches.

The second stage FB-EVAR procedure is usually done after a minimum wait period of 1 week. However, more often, 6-8 weeks are needed, due to time delay for device manufacturing. A single-stage procedure is indicated if the patient has a symptomatic or rapidly expanding TAAA. If there is suspicion of spinal cord ischemia by intraoperative neuromonitoring, the strategy of TASP may be utilized.

\section{Technical aspects}

The procedure is performed under general anesthesia in a hybrid operating room with fixed imaging. Neuromonitoring is used routinely. Patients are positioned supine with both arms raised overhead and the right axilla prepped for possible brachial access (Figure 8). Bilateral percutaneous femoral access is obtained using the preclosure technique whenever possible. Open surgical exposure of the femoral arteries is performed in less than $10 \%$ of patients because of small calcified arteries or a high femoral bifurcation.
Temporary iliac or femoral conduits may be used to facilitate early pelvic and lower limb perfusion in complex cases when prolonged operative time is expected. An iliofemoral bypass as a permanent surgical conduit is considered in patients with small or diseased iliac arteries.

Most TAAA devices are designed with a preloaded system. Our technique continues to evolve. Although we have used brachial access extensively and previously advocated this to facilitate the procedure and minimize lower extremity ischemia, currently, most TAAA patients are treated using total transfemoral approach with steerable sheaths-and without brachial access. If brachial access is used, brachial-femoral access is initially established using a long, 0.035-inch Metro guidewire (Cook Medical Inc., Bloomington IN), which is introduced from the femoral sheath and snared via the brachial approach. This helps stabilize the main delivery system and allows immediate access to the directional branches or fenestrations using the preloaded guidewires. The fenestrated-branched endovascular stent graft is deployed in a staggered fashion, starting with the celiac artery and the superior mesenteric artery, which are accessed from the brachial approach. Following placement of 0.035 -inch Amplatz Super Stiff ${ }^{\mathrm{TM}}$ guidewires (Boston Scientific, Bloomington MN) into the mesenteric vessels, the distal portion of the device is deployed, and the renal arteries are cannulated through the fenestrations (via femoral access) or branches (via brachial access) using the preloaded guidewire system. Each of the target vessels is sequentially incorporated by placement of bridging stent grafts. Most often, balloon expandable covered stents are used for fenestrations and self-expandable stent grafts are used for branches. The repair is extended distally using a universal bifurcated device and iliac limbs. Iliac branch devices may be needed in patients with concomitant iliac aneurysms.

The total transfemoral approach uses a steerable sheath with locking 0.014-inch wire to prevent the sheath from unforming and to allow sufficient support for advancement of bridging stents. The technique has been applied with four directional branches or with a combination of fenestrations and directional branches (Figure 9). Regardless of the specific device and technique, a final rotational CBCT with rotational digital subtraction angiogram is obtained to evaluate presence of endoleak or technical problems such as stent compression, vessel dissection or thrombosis. At completion, the sheaths are removed and the brachial access is closed surgically, whereas the femoral arteries are closed using a percutaneous technique. 
A

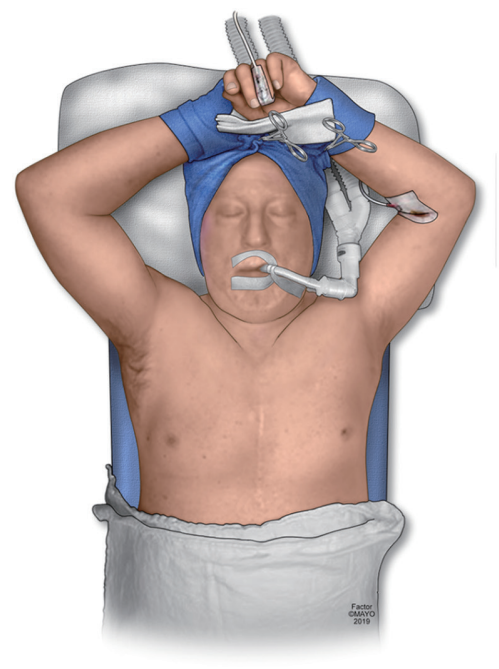

B

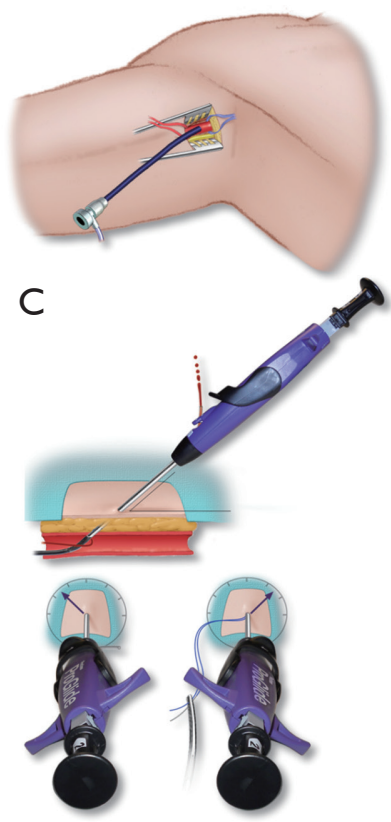

D

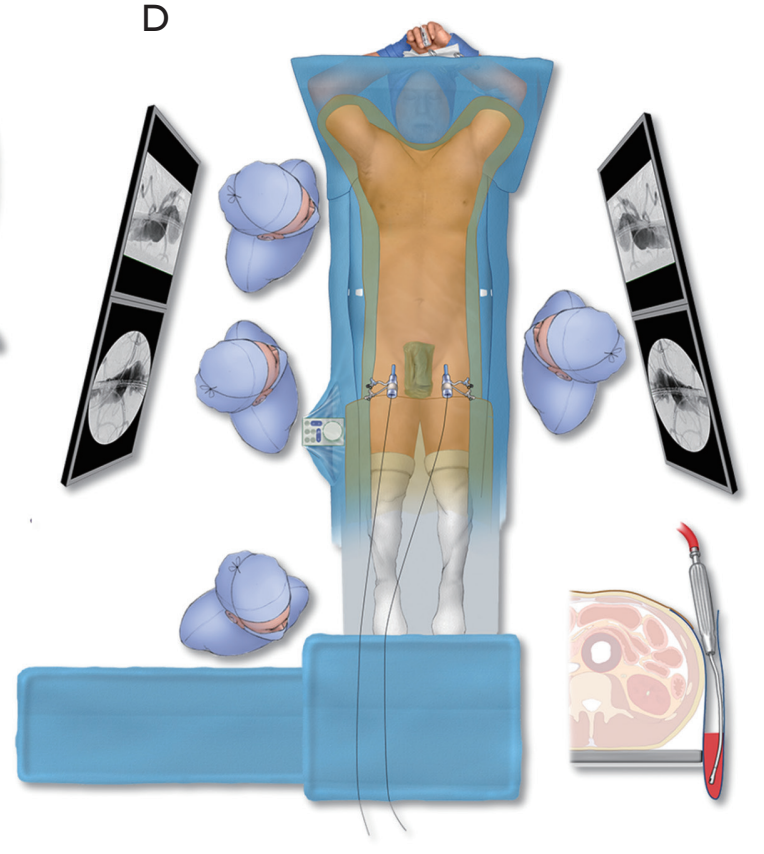

Figure 8 The patient is positioned supine with both upper extremities raised above the head for improved visualization on lateral and oblique views (A). The right brachial artery is surgically exposed in the anterior axillary line (B). Bilateral percutaneous femoral access is obtained using pre-closure technique (C). The chest, abdomen and both thighs are prepped and draped in sterile fashion. The primary operator, first assistant and a scrub nurse work on the right side of the table while the second assistant works on the left side of the table (D).

\section{Open vs. endovascular repair what is the evidence?}

The primary goal of aneurysm repair is prevention of aortic-related death without major disability. Aortic-related mortality includes death, rupture or other aortic events or mortality related to the index procedure or a secondary intervention. Other important outcomes are individual rates of disabling events, such as paraplegia, major stroke or permanent dialysis. Finally, durability of the repair should be assessed by freedom from aortic-related mortality and any secondary interventions. The latter should account for any non-aortic procedures performed to deal with operative complications or access-related problems, along with any aortic-related procedures.

There are few comparative studies directly assessing the efficacy of open surgical or endovascular TAAA repair (30-32). Limitations of the published literature include lack of granularity or standardization, inclusion of heterogeneous groups (e.g., descending thoracic aneurysms with TAAAs) and lack of detailed accounts of non-aortic and aortic reinterventions. In general, endovascular series tend to provide a more meticulous longitudinal follow up, in part because this is a requirement for endovascular techniques, and several studies and prospective IDEs designed under specific FDA regulations. Finally, it is important to consider that open surgical reports represent a mature technique performed in thousands of patients, whereas endovascular series include smaller groups, and are subject to the learning curves of the physician and the center, as well as changes in device design and techniques.

Two systematic reviews and meta-analyses have concisely summarized the literature and evidence for open surgical repair, and open $v s$. endovascular repair $(9,33)$. A metaanalysis of single-center, single-arm studies assessing paraparesis and paraplegia outcomes for open TAAA repair reported incidences ranging from $2-11 \%$ and $0-10 \%$, respectively (33). The study included 9,963 patients reported in thirty publications. The largest results stemmed from the near three decades' experience of Coselli and colleagues $(34,35)$. Although in-hospital mortality was reported in all studies to be a pooled mortality rate of $11 \%$ (95\% confidence interval, 9.56-13.09\%), other outcomes, such as renal impairment requiring permanent dialysis, stroke and tracheostomy, were reported by less than half of 

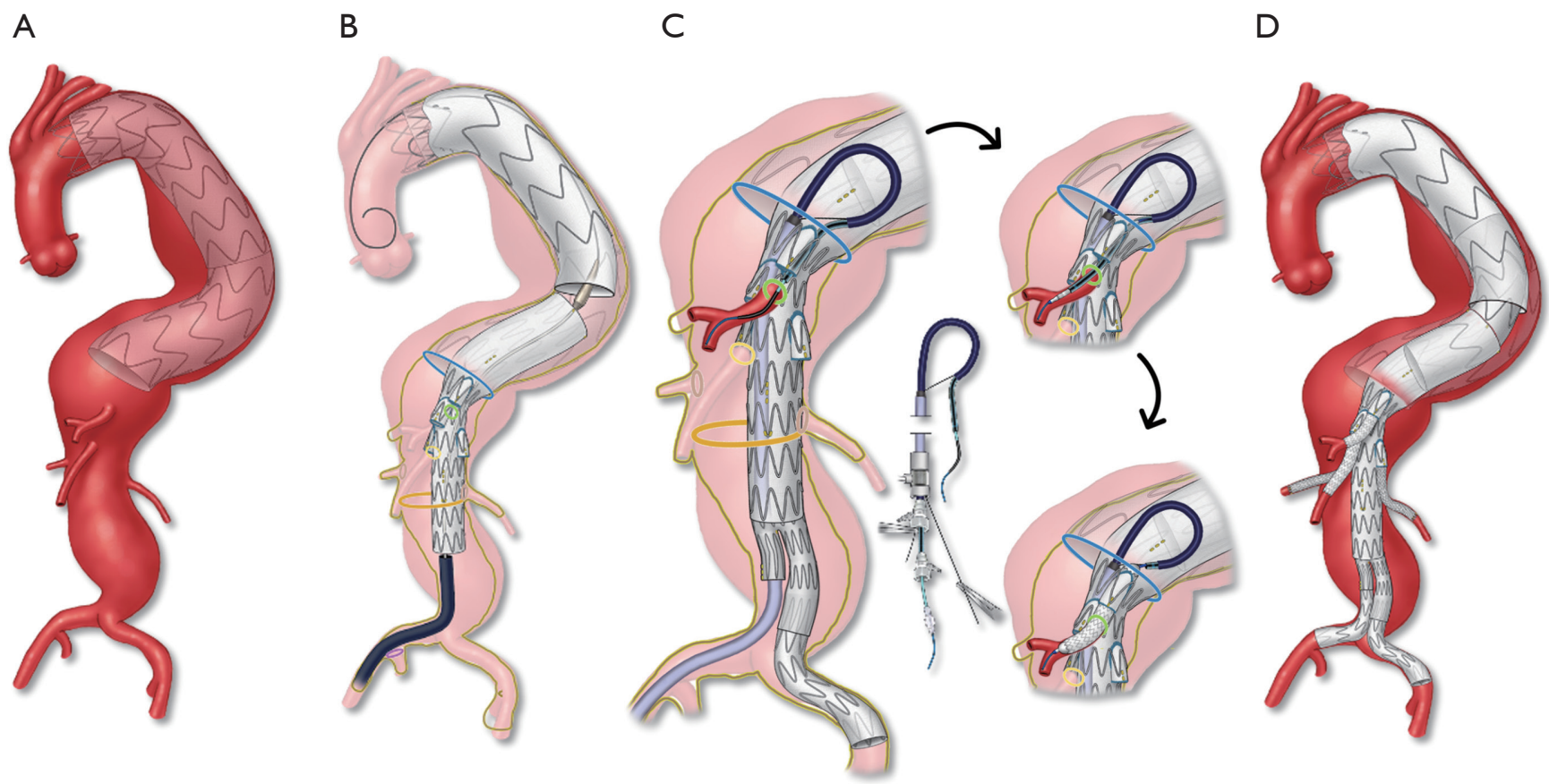

Figure 9 A first-stage TEVAR is done from zone 3 to zone 5 (A). The multibranched stent graft is deployed (B). Using total transfemoral approach and a steerable sheath that combines a $12 \mathrm{Fr}$ and $14 \mathrm{Fr}$ sheath with 0.014 -inch wire to create the support system, each of the target vessels is catheterized and stented via the femoral approach (C). The repair is completed with placement of distal bifurcated device and iliac limb extensions (D).

all studies. In this analysis, permanent dialysis was required in $8 \%$ of open surgical patients. Mortality rates were highest for Crawford Extent II TAAAs (10.3\%) and lowest for Extent I TAAAs (7.0\%). The overall patient survival at five years was $69.3 \%$. Using meta-regression analysis, the authors demonstrated that lower procedural mortality was significantly associated with higher case volume (33).

A second meta-analysis by Rocha and colleagues comparing open and endovascular TAAA repair showed lower rates of spinal cord ischemia (risk ratio, $0.65 ; 95 \%$ CI, 0.42-1.01; $\mathrm{P}=0.05$ ) and renal impairment requiring dialysis (risk ratio, 0.44; 95\% CI, 0.23-0.85; $\mathrm{P}=0.01$ ) with endovascular approach when compared to open repair (9). This meta-analysis only included comparative studies with double-arm cohorts, with a total of eight studies meeting the authors' inclusion criteria. However, inconsistent reporting of outcomes across the studies coupled with an observational design of these studies limits the ability to draw meaningful conclusions from this analysis. Although mortality, stroke, paraplegia and dialysis are widely used outcomes, none of the studies in the meta-analysis reported these end-points individually (9). Nevertheless, the study highlights differences in patient selection, with older and higher risk patients treated predominantly by endovascular approach.

From the articles included in this meta-analysis, a comparative study of 879 TAAA repairs by Locham and colleagues described higher in-hospital mortality rates and longer length of hospital stay after open surgical vs. endovascular repair $(15 \%$ vs. $5 \%, \mathrm{P}<0.001)$ (32). In the study by Ferrer and colleagues, there was no significant difference in thirty-day mortality and paraplegia between endovascular and open matched pairs. Rates of survival at two years were similar $(82.8 \%$ vs. $84.9 \%, \mathrm{P}=0.9)$, as were rates of freedom from intervention at twenty-four months $(91.0 \%$ vs. $89.7 \%, \mathrm{P}=0.3$ ) (31). In none of the aforementioned comparative studies were rates of secondary interventions reported in detail. Early procedures, such as reoperations for bleeding, dialysis access, tracheostomy or other woundrelated problems were typically not considered secondary interventions. Moreover, none of the studies reported the overall freedom from aortic-related death, which is a timedependent outcome that includes thirty-day postoperative mortality and other procedures or aortic deaths that occur beyond the first thirty days.

The majority of experience with open TAAA repair has 
been described by several large series originating from a handful of pioneering institutions. The lifelong experience of E. Stanley Crawford was reported by Svensson and colleagues, including 1,509 consecutive patients treated by open TAAA repair between 1960 and 1991 (36). The 30 -day mortality was $8 \%$ and the incidence of spinal cord ischemia, including paraplegia and paraparesis, was $16 \%$. Dialysis was required in 9\% of the patients. In 2016, Coselli and associates published the largest worldwide single-center experience with open TAAA repair in 3,309 patients (34). The thirty-day mortality was $7.5 \%$ and the rate of any permanent spinal cord injury was $5.4 \%$, with as low as $2.9 \%$ permanent paraplegia rate. Based on the extent of the aneurysmal disease, the rates of paraplegia substantially improved compared to the original report of Crawford's experience, from $15 \%$ to $1 \%$ for Extent I, $31 \%$ to $5 \%$ for Extent II, 7\% to 4\% for Extent III and 4\% to 1\% for Extent IV TAAAs $(34,36)$. A series of 1,273 open TAAA repairs reported by Estrera and colleagues in 2015 had a thirty-day mortality of $16 \%$ and a five-year survival rate of $60 \%$ (37). Other single-center studies reported relatively low mortality for open TAAA repair. Tanaka and colleagues (38) reported on 100 patients treated by open TAAA repair, with an inhospital mortality of $5 \%$. Latz and colleagues reported a thirty-day mortality of $8 \%$ among 506 patients treated for Extent I to III TAAAs (39). Girardi and colleagues reported a thirty-day mortality of $5.6 \%$ amongst 783 patients, but this included 246 patients who had descending thoracic aneurysms (40). Review of national datasets, such as the National Surgical Quality Improvement Program (NSQIP) database (41), show more ominous outcomes, with perioperative mortality after TAAA open repair being $14.0 \%$ (non-ruptured $11.4 \%$ vs. ruptured $34.2 \%, \mathrm{P}<0.01$ ).

A fundamental difference between open surgical and endovascular series relies on patient selection. Almost all open surgical series include patients with an average age of 50 to 65 years, which is, on average, a decade younger than endovascular series. Rates of cardiovascular risk factors and severity scores are significantly higher in patients treated by endovascular approach, particularly the rates of severe cardiac, pulmonary and renal disease. In general, more than two-thirds of the patients treated by endovascular repair are considered high risk or prohibitively high risk for open surgical repair. Conversely, open surgical reports generally include a higher proportion of patients with chronic postdissection aneurysms and genetically triggered aortic diseases. Despite these fundamental differences in patient selection and the effect of the learning curve, outcomes are similar or better for endovascular repair (Tables 1,2). Recent contemporary reports consistently show in-hospital mortalities for FB-EVAR in the range of $0.6 \%$ to $5 \%$ for Extent I to III TAAAs $(53,56,59)$ and $1 \%$ to $1.5 \%$ for Extent IV or complex abdominal aortic aneurysms $(53,54)$. Oderich and associates reported 430 consecutive patients treated by FB-EVAR in a prospective PS-IDE study, including 165 patients with Extent I to III and 132 patients with Extent IV TAAAs. In that report, thirty-day mortality was $0.6 \%$ for Extent I to III and $1.5 \%$ for Extent IV TAAAs (53). More recently, the United States Aortic Research Consortium (US ARC) has analyzed the data of 1,681 consecutive patients from nine PS-IDE studies. In that analysis, overall mortality was $2 \%$ for Extent IV and for Extent I-III TAAAs (63).

Although the safety of endovascular TAAA repair may be supported by recent series, the main criticism has been the high rate of secondary interventions. Few reports have shown outcomes beyond one to two years, and there are no comparisons with open surgical repair. Coselli and associates (34) reported a freedom from late aortic-related procedure of $94.1 \%$ at fifteen years. However, only 189 of the 3,309 patients initially treated by open surgical repair were included in the analysis. The Massachusetts General Hospital group (39) reported rates of freedom from secondary aortic interventions at one year to be $96 \%, 84 \%$ at five years, and $75 \%$ at ten years. However, none of these studies have shown freedom from any type of reintervention. Huang and colleagues (48) have analyzed 287 non-emergency patients treated by open or endovascular TAAA repair at the Mayo Clinic between 2007 and 2015. In this study, freedom from any type of reintervention at two years was similar for open surgical repair $(68 \%)$ and FB-EVAR $(63 \%)$.

Surgeon and hospital volume are directly related to outcomes after high-complexity procedures, such as esophagectomy, Whipple operation, open and endovascular TAAA repair $(33,64)$. These procedures should be ideally performed in high-volume 'centers of excellence'. With respect to FB-EVAR, not only should the center have clinical expertise from vascular surgery, cardiac surgery, cardiovascular anesthesia and critical care, it is important that the team master complex endovascular skills-and that the center is equipped with a hybrid operating room, advanced imaging and access to a wide endovascular inventory. Given the association of clinical outcomes with surgeon and hospital volume, it is important that minimum case thresholds be defined. Using cumulative sum methodology, our group demonstrated that a minimum of 


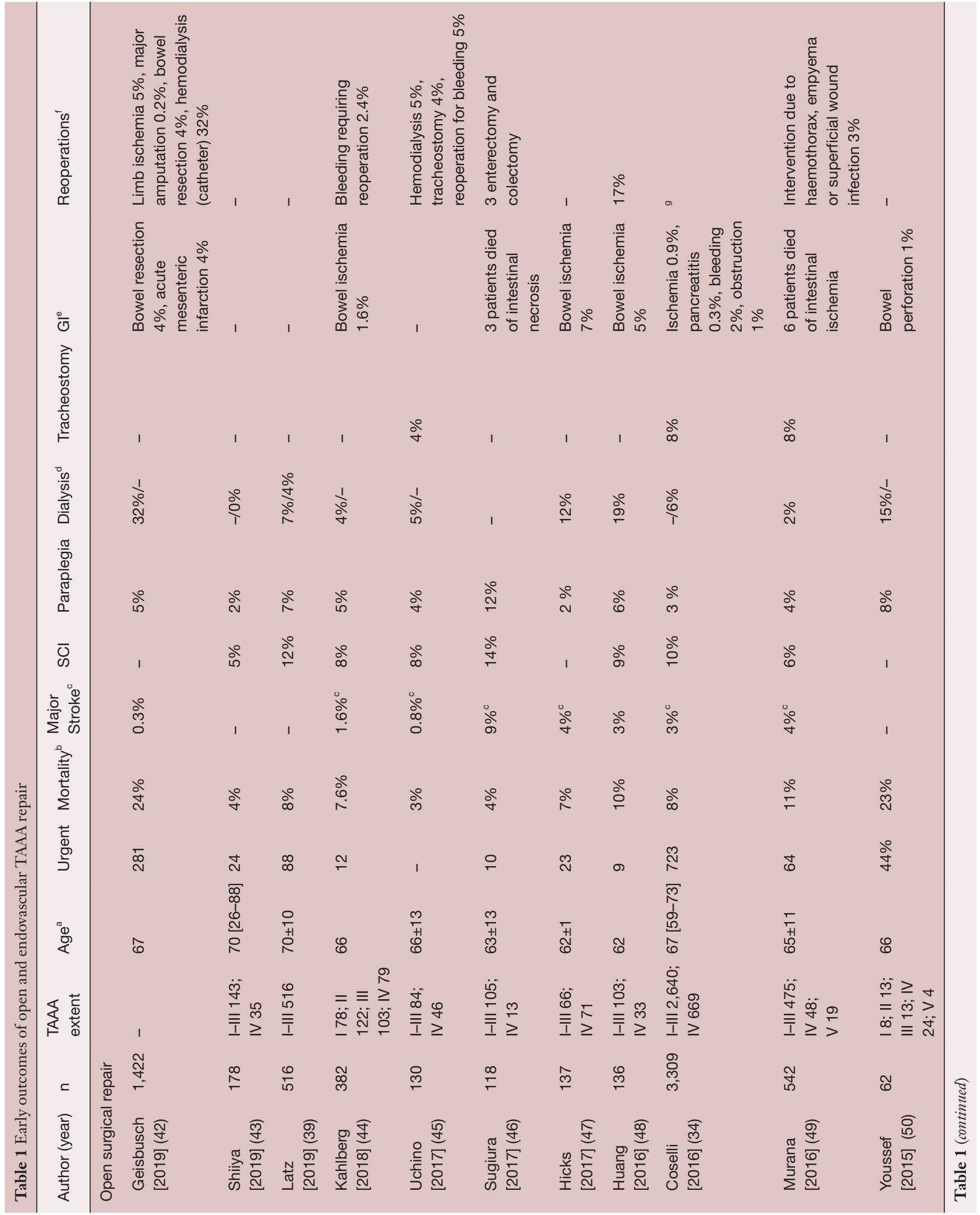




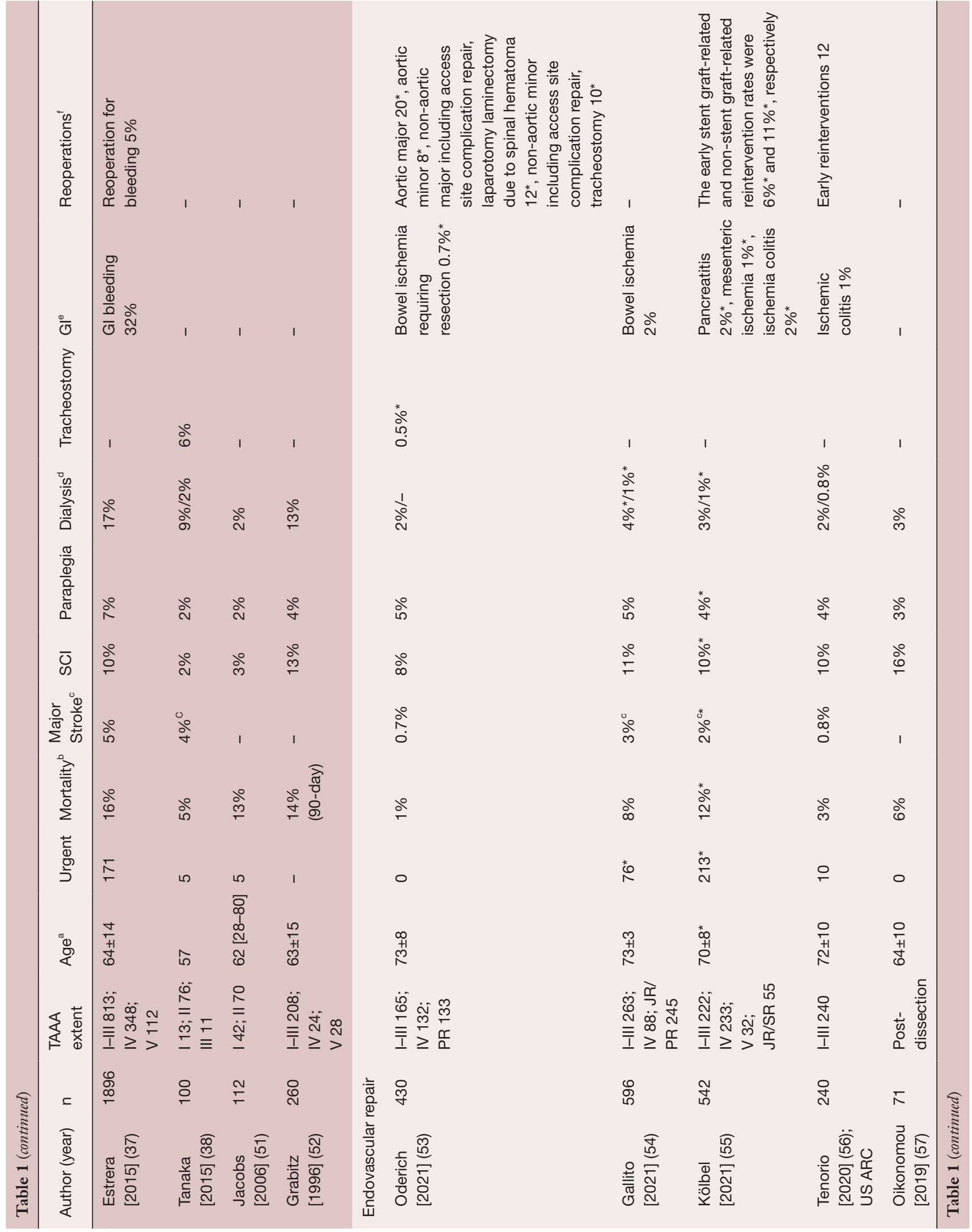




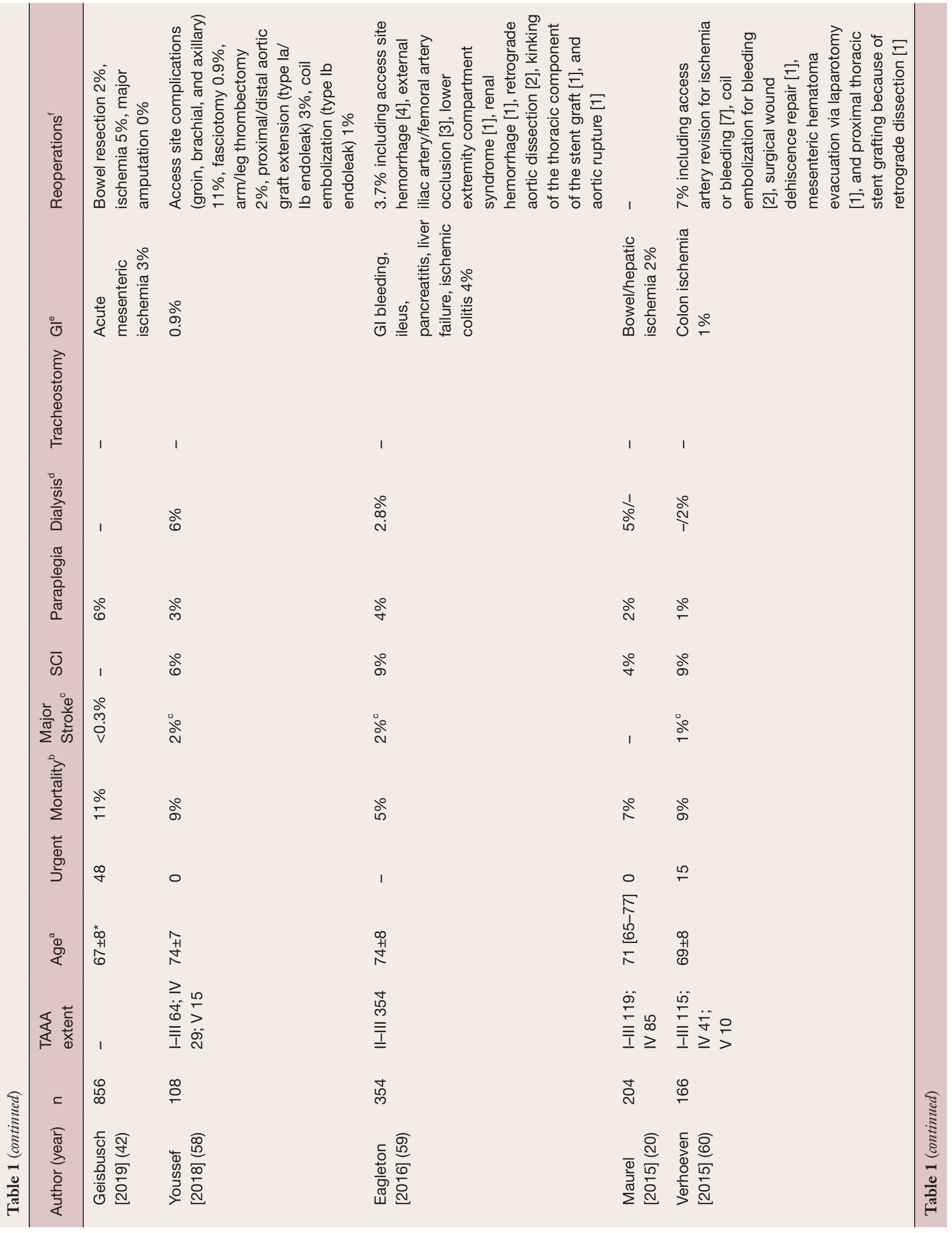




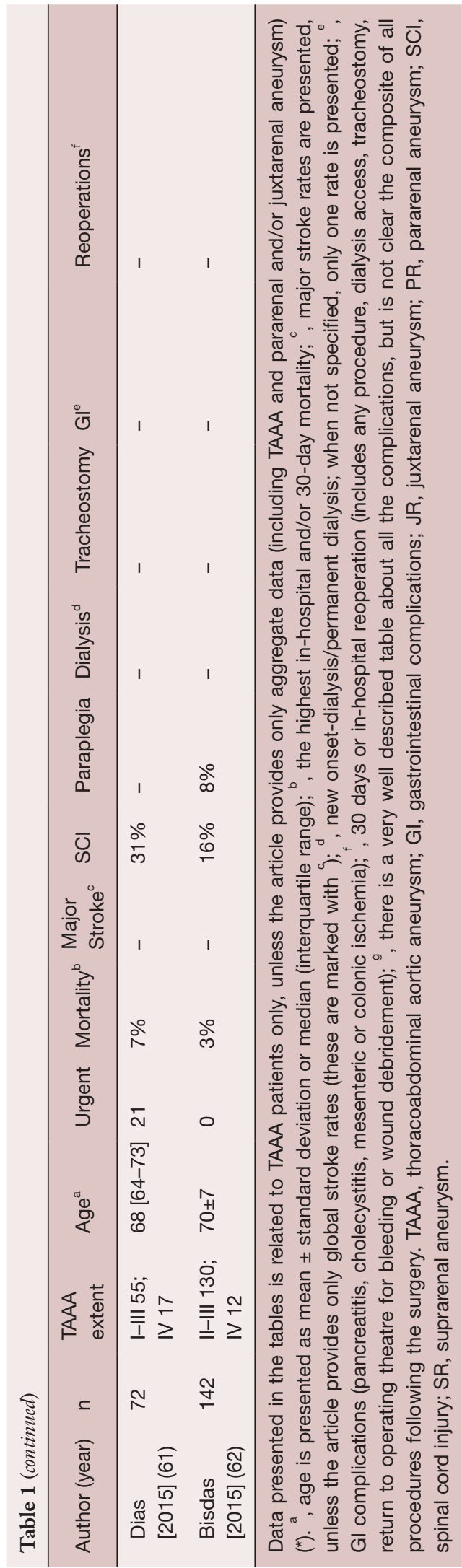

thirty-two FB-EVAR procedures per year were needed to achieve low event rates, with improved outcomes after one hundred consecutive cases (65).

\section{Patient selection for endovascular TAAA repair}

Treatment selection varies depending on access to endovascular technology, which is currently limited in the US. In Germany, a nationwide survey from 2005 to 2014 demonstrated a shift from open surgical to endovascular repair as the most frequent treatment modality for TAAA (42). In that study, up to $90 \%$ of descending thoracic aneurysms and $75 \%$ of TAAAs were treated by endovascular approach in the last year of the survey. Other studies have shown a doubling in the number of TAAA repairs in the last decade, largely due to the introduction of less-invasive endovascular techniques. The potential advantages of early survival benefit, decreased risk of complications and faster recovery, allowed treatment of older, frail and higher risk patients who were previously not considered suitable candidates for open surgery (66). However, these complex endovascular procedures are not without risk of complications, and there is a need for continued surveillance and potential for secondary interventions (31). The impact of the repair on the patient's quality of life should be carefully balanced against the benefit of preventing aneurysm related mortality. Often, in elderly or higher risk patients, the decision of whether to operate is not 'can you do it' but rather 'should you do it'.

The indication for endovascular repair is the same as that for open surgical repair. Aside from risk stratification and anticipated survival, anatomical factors remain the most important eligibility criteria for suitability to endovascular TAAA repair. A thorough preoperative evaluation should include cardiac, renal and pulmonary risk assessments. American Society of Anesthesiologists (ASA) classification score of 4 is associated with a significantly higher early- and late-mortality rates $(67,68)$. We typically do not recommend FB-EVAR in patients with a life expectancy of less than two years. Patients with advanced malignancy, severe congestive heart failure or GOLD 4 chronic obstructive pulmonary disease are usually managed conservatively. Although advanced age should be carefully considered, physiologic age is usually more important (69). Motta and colleagues, through analyzing the US ARC data, showed that FB-EVAR was safe in octogenarians, with a thirtyday mortality of $0.5 \%$. However, octogenarians had lower survival at three years compared with non-octogenarians (70). 


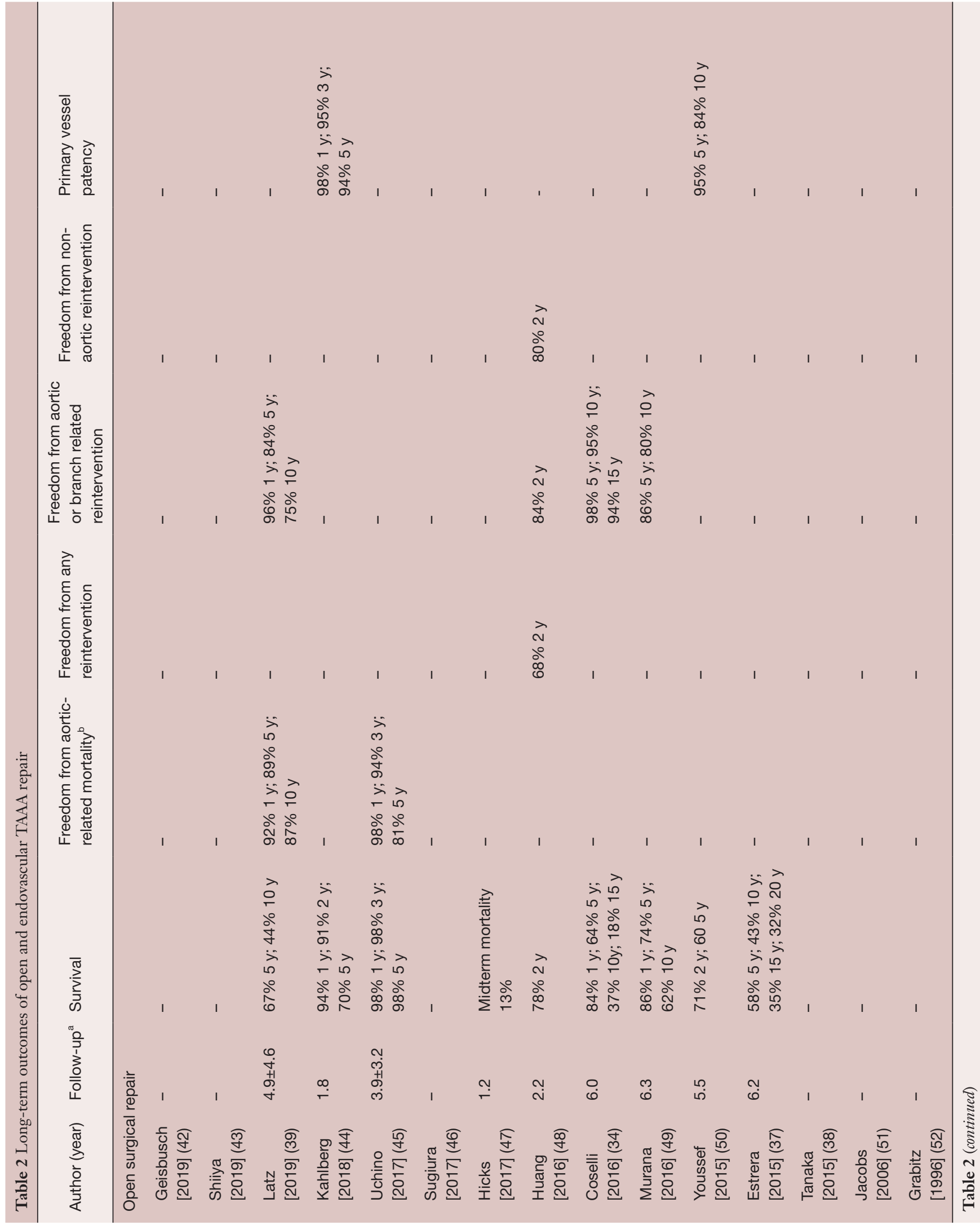




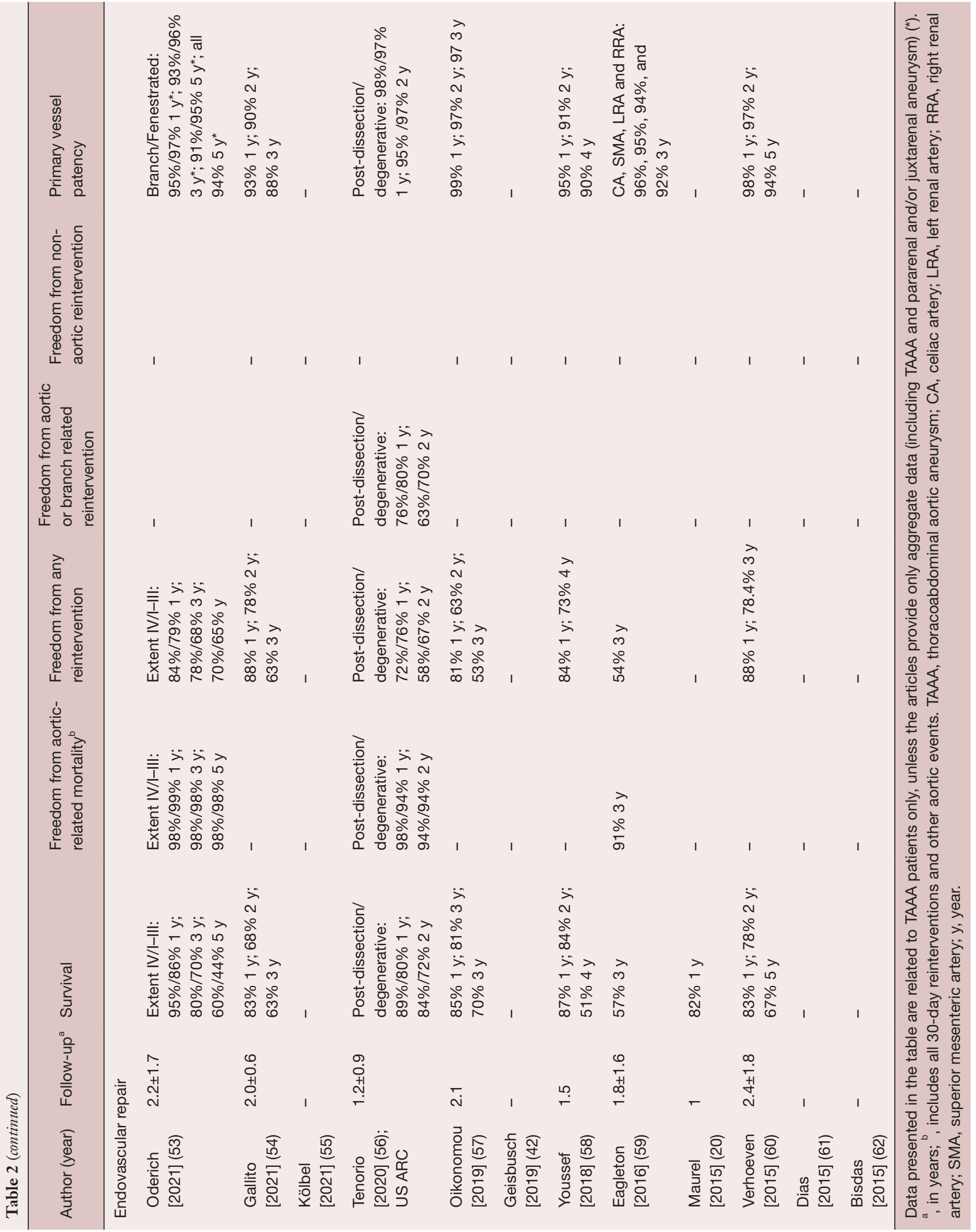


Assessment of frailty may be important in decisionmaking, as we evaluate higher risk subgroups, such as elderly or deconditioned patients (71). There is a clinical need for development of an assessment tool that complements the traditional preoperative evaluation to predict survival after endovascular TAAA repair. Several multidomain and singledomain assessment tools have been studied in patients undergoing vascular surgery (72). Assessment of frailty using functional status has been shown to predict shortterm mortality in elderly patients after vascular surgical procedures (73). In addition, central muscle mass, as a surrogate for sarcopenia, may help determine long-term survival prognosis in patients undergoing vascular surgical and endovascular procedures $(74,75)$. This is usually expressed as psoas muscle area measured at the L3/4 level on axial computed tomography images. However, these tools have not been validated specifically for endovascular repair of complex aneurysms, such as TAAAs, and although some have been successful in showing the association of psoas muscle area with mid- to long-term mortality (76), others have failed to replicate these results (77).

Conservative treatment is a reasonable option for some TAAA patients. Life expectancy of patients with large untreated TAAAs is relatively short. In 1986, Crawford and DeNatale (78) published an observational study of patients who were denied or refused open TAAA repair. Only $24 \%$ of patients were alive at the two-year follow up. Half of the deaths were attributed to aneurysm rupture. In a Mayo Clinic cohort study of 57 patients with TAAAs who were initially managed nonoperatively, fifteen underwent subsequent repair and eight developed aneurysm rupture, accounting for $24 \%$ of all deaths during the follow-up period (79). The five-year, repair-free survival was $17 \%$. In 2010, Hansen and co-authors reported 64\% survival at oneyear and $52 \%$ at two years among 89 TAAA patients who were denied treatment (80). Nearly all of these patients died within five years-aneurysm rupture accounted for half of these deaths.

Health-related quality of life outcomes have been extensively investigated for infrarenal endovascular aortic repair in several randomized controlled trials and in two meta-analyses $(81,82)$. These studies showed better quality of life metrics for endovascular repair compared to open surgical repair at one-year, but similar results beyond two years. Regarding endovascular repair of TAAAs, there is limited data for endovascular repair and no longitudinal data for open surgical repair. Our group published a prospective series of 159 consecutive patients undergoing fenestrated- branched endovascular repair, including 102 patients with TAAAs (83). In that study, patients with TAAAs had worse baseline physical component scores, which were especially low among patients who had undergone previous firststage endovascular repair and even lower in those who had prior open surgical repair. Physical component scores were lower at baseline in all patients with TAAAs, irrespective of prior aortic repair, which suggests that extensive aneurysmal disease is associated with a negative impact on quality of life, independent of repair. Following FB-EVAR, there was a decline in physical quality of life measures at six-eight weeks, which returned to baseline at six-twelve months in patients with pararenal aneurysms, but not in those with TAAAs. Therefore, elective endovascular TAAA repair has a significant impact on patients' physical component scores that lasts at least one year after repair, and this should be considered when discussing treatment options.

\section{Limitations of endovascular TAAA repair}

Endovascular repair has not been evaluated in pivotal studies to treat patients with connective tissue disease. In this group, there is concern about tissue fragility, risk of retrograde dissections and progressive aneurysm degeneration compromising the seal. The Society of Thoracic Surgeons and European Society for Vascular Surgery guidelines on diagnosis and treatment of aortic diseases recommends against endovascular repair in patients with connective tissue disease, unless operative risk has been deemed prohibitive or in emergency situations $(8,84)$. There are potential exceptions in which endovascular repair may have an increasing role. Patients who failed prior open arch and TAAA repair-and have proximal surgical grafts that serve as landing zones-may be considered for endovascular repair. Endovascular repair may be used in patients with ruptured aneurysms as a bridge to open surgical repair (85). Elective open surgical repair has also been associated with significant risk of morbidity and mortality among patients with a connective tissue disorder. A recent study of 65 patients with a mean age of 56 years and a diagnosis of Marfan's syndrome showed perioperative mortality of $14 \%$, with a $15 \%$ rate of early reinterventions (86).

Anatomical considerations that may limit the indication of an endovascular approach include excessive aortic debris in the aortic arch, descending aorta and visceral segment (e.g., shaggy aorta). Branch incorporation involves significant catheter manipulation that can easily dislodge loose debris into the supra-aortic branches, visceral, 
intercostal vessels and lower extremity arteries, potentially causing catastrophic consequences (87). In addition, severe tortuosity and angulation of the aorta can make the passage and orientation of endovascular devices difficult, resulting in misalignment of fenestrations and branches. This can sometimes be overcome with the use of a brachialto-femoral application system with enough tension on the through-and-through wire. Narrow and calcified or occluded iliac arteries may also be a limiting factor. However, many access difficulties can often be overcome with the use of surgical or endovascular conduits (88). Open surgical conduits are performed either prior to the procedure (permanent iliofemoral conduit) or in conjunction with the endovascular repair. Our preference is to use a $12 \mathrm{~mm}$ polyester graft anastomosed in an end-toside fashion. This graft diameter allows the introduction of a $24 \mathrm{Fr}$ delivery system without difficulty. The endoconduit, or 'paving and crack' technique, is an alternative that applies the use of covered stents from the common iliac artery to the distal external iliac artery, with subsequent forceful dilatation to enlarge the vessel to 10 or $12 \mathrm{~mm}$ (89). Because this technique requires coverage of the internal iliac artery and may reduce the collateral flow of the spinal cord, we do not recommend its use in patients with previously patent internal iliac arteries who are planned for extensive TAAA repair. Finally, the presence of suitable target vessels without excessive tortuosity, occlusive disease or early bifurcation is critical for successful endovascular branch incorporation.

\section{Conclusions}

FB-EVAR offers a minimally invasive approach to open TAAA repair, which is the largest and most morbid procedure of our specialty. The decision between open or endovascular technique should take into consideration the outcomes at each individual institution as well as the suitability of each approach for a prospective patient. Outcomes of open surgical repair remain stagnant in most centers, and the lowest mortality (7.5\%) has been achieved only after three decades of experience with more than 3,500 patients. Conversely, lower mortality rates have already been reported in several centers with endovascular techniques applied to older and higher risk patients. In some series, mortalities have been consistently lower than $4 \%$ for Extent I and II TAAAs. Therefore, in most centers with mature programs and access to fenestrated-branched technology, endovascular repair has become the new gold standard-and is the first treatment option in patients who have suitable anatomy and do not have connective tissue disorders or aortic infections, independent of their clinical risk or age. Open surgical repair should be performed in selected centers of excellence and is primarily indicated in younger patients with connective tissue disorders, in those with aortic infections and in patients with unsuitable anatomy for endovascular approach. It is likely that continued evolution of patient selection, device design and improved perioperative care will continue to decrease the already lower mortality and morbidity of endovascular repair. Universally adopted reporting standards for patient characteristics, outcomes, and the conduct of contemporary comparative studies will allow for better assessment and comparisons of the risks associated with the two surgical treatment options for TAAA.

\section{Acknowledgments}

Funding: None.

\section{Footnote}

Conflicts of Interest: ALE is a consultant for W.L. Gore. GSO is a consultant for Cook Medical, W. L. Gore, Centerline Biomedical and GE Healthcare (all paid to Mayo Clinic and University of Texas Health Science at Houston, with no personal income). The other authors have no conflicts of interest to declare.

Open Access Statement: This is an Open Access article distributed in accordance with the Creative Commons Attribution-NonCommercial-NoDerivs 4.0 International License (CC BY-NC-ND 4.0), which permits the noncommercial replication and distribution of the article with the strict proviso that no changes or edits are made and the original work is properly cited (including links to both the formal publication through the relevant DOI and the license). See: https://creativecommons.org/licenses/by-nc-nd/4.0/.

\section{References}

1. Ziganshin BA, Elefteriades JA. Surgical management of thoracoabdominal aneurysms. Heart 2014;100:1577-82.

2. Juvonen T, Ergin MA, Galla JD, et al. Prospective study of the natural history of thoracic aortic aneurysms. Ann Thorac Surg 1997;63:1533-45.

3. Crawford ES. Thoraco-abdominal and abdominal aortic aneurysms involving renal, superior mesenteric, celiac 
arteries. Ann Surg 1974;179:763-72.

4. Svensson LG, Hess KR, Coselli JS, et al. Influence of segmental arteries, extent, and atriofemoral bypass on postoperative paraplegia after thoracoabdominal aortic operations. J Vasc Surg 1994;20:255-62.

5. Frederick JR, Woo YJ. Thoracoabdominal aortic aneurysm. Ann Cardiothorac Surg 2012;1:277-85.

6. Chuter TA, Gordon RL, Reilly LM, et al. An endovascular system for thoracoabdominal aortic aneurysm repair. J Endovasc Ther 2001;8:25-33.

7. Oderich GS, Ribeiro MS, Sandri GA, et al. Evolution from physician-modified to company-manufactured fenestrated-branched endografts to treat pararenal and thoracoabdominal aortic aneurysms. J Vasc Surg 2019;70:31-42.e7.

8. Wanhainen A, Verzini F, Van Herzeele I, et al. Editor's Choice - European Society for Vascular Surgery (ESVS) 2019 Clinical Practice Guidelines on the Management of Abdominal Aorto-iliac Artery Aneurysms. Eur J Vasc Endovasc Surg 2019;57:8-93.

9. Rocha RV, Friedrich JO, Elbatarny M, et al. A systematic review and meta-analysis of early outcomes after endovascular versus open repair of thoracoabdominal aortic aneurysms. J Vasc Surg 2018;68:1936-1945.e5.

10. Rocha RV, Lindsay TF, Friedrich JO, et al. Systematic review of contemporary outcomes of endovascular and open thoracoabdominal aortic aneurysm repair. J Vasc Surg 2020;71:1396-1412.e12.

11. Mirza AK, Tenorio ER, Kärkkäinen JM, et al. Outcomes of a novel upper extremity preloaded delivery system for fenestrated-branched endovascular repair of thoracoabdominal aneurysms. J Vasc Surg 2020;72:470-9.

12. Mirza AK, Tenorio ER, Kärkkäinen JM, et al. Technical video of endovascular repair of chronic postdissection thoracoabdominal aortic aneurysm using a five-vessel preloaded fenestrated-branched stent graft. J Vasc Surg 2019;69:296-302.e1.

13. Oderich GS, Farber MA, Silveira PG, et al. Technical aspects and 30-day outcomes of the prospective early feasibility study of the GORE EXCLUDER Thoracoabdominal Branched Endoprosthesis (TAMBE) to treat pararenal and extent IV thoracoabdominal aortic aneurysms. J Vasc Surg 2019;70:358-368.e6.

14. Tenorio ER, Oderich GS, Sandri GA, et al. Impact of onlay fusion and cone beam computed tomography on radiation exposure and technical assessment of fenestratedbranched endovascular aortic repair. J Vasc Surg 2019;69:1045-1058.e3.
15. Tenorio ER, Oderich GS, Sandri GA, et al. Prospective nonrandomized study to evaluate cone beam computed tomography for technical assessment of standard and complex endovascular aortic repair. J Vasc Surg 2020;71:1982-1993.e5.

16. Mendes BC, Oderich GS, Reis de Souza L, et al. Implications of renal artery anatomy for endovascular repair using fenestrated, branched, or parallel stent graft techniques. J Vasc Surg 2016;63:1163-1169.e1.

17. Mendes BC, Oderich GS. Endovascular repair of thoracoabdominal aortic aneurysm using the off-theshelf multibranched t-Branch stent graft. J Vasc Surg 2016;63:1394-1399.e2.

18. Chuter TA, Hiramoto JS, Park KH, et al. The transition from custom-made to standardized multibranched thoracoabdominal aortic stent grafts. J Vasc Surg 2011;54:660-7; discussion 667-8.

19. Gasper WJ, Reilly LM, Rapp JH, et al. Assessing the anatomic applicability of the multibranched endovascular repair of thoracoabdominal aortic aneurysm technique. J Vasc Surg 2013;57:1553-8; discussion 1558.

20. Maurel B, Delclaux N, Sobocinski J, et al. The impact of early pelvic and lower limb reperfusion and attentive peri-operative management on the incidence of spinal cord ischemia during thoracoabdominal aortic aneurysm endovascular repair. Eur J Vasc Endovasc Surg 2015;49:248-54.

21. Eagleton MJ, Shah S, Petkosevek D, et al. Hypogastric and subclavian artery patency affects onset and recovery of spinal cord ischemia associated with aortic endografting. J Vasc Surg 2014;59:89-94.

22. Tanaka H, Minatoya K, Matsuda H, et al. Embolism is emerging as a major cause of spinal cord injury after descending and thoracoabdominal aortic repair with a contemporary approach: magnetic resonance findings of spinal cord injury. Interact Cardiovasc Thorac Surg 2014;19:205-10.

23. Etz CD, Kari FA, Mueller CS, et al. The collateral network concept: remodeling of the arterial collateral network after experimental segmental artery sacrifice. J Thorac Cardiovasc Surg 2011;141:1029-36.

24. Bischoff MS, Scheumann J, Brenner RM, et al. Staged approach prevents spinal cord injury in hybrid surgicalendovascular thoracoabdominal aortic aneurysm repair: an experimental model. Ann Thorac Surg 2011;92:138-46; discussion 146.

25. O'Callaghan A, Mastracci TM, Eagleton MJ. Staged endovascular repair of thoracoabdominal aortic aneurysms 
limits incidence and severity of spinal cord ischemia. J Vasc Surg 2015;61:347-354.e1.

26. Bischoff MS, Brenner RM, Scheumann J, et al. Staged approach for spinal cord protection in hybrid thoracoabdominal aortic aneurysm repair. Ann Cardiothorac Surg 2012;1:325-8.

27. Branzan D, Etz CD, Moche M, et al. Ischaemic preconditioning of the spinal cord to prevent spinal cord ischaemia during endovascular repair of thoracoabdominal aortic aneurysm: first clinical experience. EuroIntervention 2018;14:828-35.

28. Sonesson B, Dias NV, Resch TA. New Technique for Preconditioning of the Spinal Cord Before Endovascular Repair of Descending Thoracic and Thoracoabdominal Aortic Aneurysms. J Endovasc Ther 2019;26:691-6.

29. Kawajiri H, Tenorio ER, Khasawneh MA, et al. Staged total arch replacement, followed by fenestrated-branched endovascular aortic repair, for patients with mega aortic syndrome. J Vasc Surg 2021;73:1488-1497.e1.

30. Bertoglio L, Mascia D, Cambiaghi T, et al. Management of visceral aortic patch aneurysms after thoracoabdominal repair with open, hybrid, or endovascular approach. J Vasc Surg 2018;67:1360-71.

31. Ferrer C, Cao P, De Rango P, et al. A propensitymatched comparison for endovascular and open repair of thoracoabdominal aortic aneurysms. J Vasc Surg 2016;63:1201-7.

32. Locham S, Dakour-Aridi H, Nejim B, et al. Outcomes and cost of open versus endovascular repair of intact thoracoabdominal aortic aneurysm. J Vasc Surg 2018;68:948-955.e1.

33. Moulakakis KG, Karaolanis G, Antonopoulos CN, et al. Open repair of thoracoabdominal aortic aneurysms in experienced centers. J Vasc Surg 2018;68:634-645.e12.

34. Coselli JS, LeMaire SA, Preventza O, et al. Outcomes of 3309 thoracoabdominal aortic aneurysm repairs. J Thorac Cardiovasc Surg 2016;151:1323-37.

35. LeMaire SA, Price MD, Green SY, et al. Results of open thoracoabdominal aortic aneurysm repair. Ann Cardiothorac Surg 2012;1:286-92.

36. Svensson LG, Crawford ES, Hess KR, et al. Experience with 1509 patients undergoing thoracoabdominal aortic operations. J Vasc Surg 1993;17:357-68; discussion 368-70.

37. Estrera AL, Sandhu HK, Charlton-Ouw KM, et al. A Quarter Century of Organ Protection in Open Thoracoabdominal Repair. Ann Surg 2015;262:660-8.

38. Tanaka H, Minatoya K, Sasaki H, et al. Recent thoracoabdominal aortic repair outcomes using moderate-to-deep hypothermia combined with targeted reconstruction of the Adamkiewicz artery†. Interact Cardiovasc Thorac Surg 2015;20:605-10; discussion 610.

39. Latz CA, Cambria RP, Patel VI, et al. Durability of open surgical repair of type I-III thoracoabdominal aortic aneurysm. J Vasc Surg 2019;70:413-23.

40. Girardi LN, Leonard JR, Lau C, et al. Gender-related outcomes after open repair of descending thoracic and thoracoabdominal aortic aneurysms. J Vasc Surg 2019;69:1028-1035.e1.

41. Obeid T, Hicks CW, Yin K, et al. Contemporary outcomes of open thoracoabdominal aneurysm repair: functional status is the strongest predictor of perioperative mortality. J Surg Res 2016;206:9-15.

42. Geisbüsch S, Kuehnl A, Salvermoser M, et al. Increasing Incidence of Thoracic Aortic Aneurysm Repair in Germany in the Endovascular Era: Secondary Data Analysis of the Nationwide German DRG Microdata. Eur J Vasc Endovasc Surg 2019;57:499-509.

43. Shiiya N, Washiyama N, Tsuda K, et al. Japanese perspective in surgery for thoracoabdominal aortic aneurysms. Gen Thorac Cardiovasc Surg 2019;67:187-91.

44. Kahlberg A, Ferrante AMR, Miloro R, et al. Late patency of reconstructed visceral arteries after open repair of thoracoabdominal aortic aneurysm. J Vasc Surg 2018;67:1017-24.

45. Uchino G, Yunoki K, Sakoda N, et al. Spinal cord protection during thoracoabdominal aortic replacement: spinal cord perfusion maintenance. Interact Cardiovasc Thorac Surg 2017;24:708-13.

46. Sugiura J, Oshima H, Abe T, et al. The efficacy and risk of cerebrospinal fluid drainage for thoracoabdominal aortic aneurysm repair: a retrospective observational comparison between drainage and non-drainage. Interact Cardiovasc Thorac Surg 2017;24:609-14.

47. Hicks CW, Lue J, Glebova NO, et al. A 10-year institutional experience with open branched graft reconstruction of aortic aneurysms in connective tissue disorders versus degenerative disease. J Vasc Surg 2017;66:1406-16.

48. Huang Y, Oderich G, Ribeiro M, et al. Treatment Trends and Outcomes of Open and Endovascular Thoracoabdominal Aortic Repairs in a Single Center. Eur J Vasc Endovasc Surg 2016;52:398.

49. Murana G, Castrovinci S, Kloppenburg G, et al. Open thoracoabdominal aortic aneurysm repair in the modern era: results from a 20-year single-centre experience. Eur J Cardiothorac Surg 2016;49:1374-81.

50. Youssef M, Neufang A, Jungmann F, et al. Patency of renal 
and visceral vessels after open thoracoabdominal aortic replacement. J Vasc Surg 2015;62:594-9.

51. Jacobs MJ, Mess W, Mochtar B, et al. The value of motor evoked potentials in reducing paraplegia during thoracoabdominal aneurysm repair. J Vasc Surg 2006;43:239-46.

52. Grabitz K, Sandmann W, Stühmeier K, et al. The risk of ischemic spinal cord injury in patients undergoing graft replacement for thoracoabdominal aortic aneurysms. J Vasc Surg 1996;23:230-40.

53. Oderich GS, Tenorio ER, Mendes BC, et al. Midterm Outcomes of a Prospective, Nonrandomized Study to Evaluate Endovascular Repair of Complex Aortic Aneurysms Using Fenestrated-Branched Endografts. Ann Surg 2021;274:491-9.

54. Gallitto E, Faggioli G, Melissano G, et al. Preoperative and postoperative predictors of clinical outcome of fenestrated and branched endovascular repair for complex abdominal and thoracoabdominal aortic aneurysms in an Italian multicenter registry. J Vasc Surg 2021. [Epub ahead of print]. doi: 10.1016/j.jvs.2021.04.072.

55. Kölbel T, Spanos K, Jama K, et al. Early outcomes of the t-Branch off-the-shelf multi-branched stent graft in 542 patients for elective and urgent aortic pathologies - a retrospective observational study. J Vasc Surg 2021. [Epub ahead of print]. doi: 10.1016/j.jvs.2021.05.041.

56. Tenorio ER, Oderich GS, Farber MA, et al. Outcomes of endovascular repair of chronic postdissection compared with degenerative thoracoabdominal aortic aneurysms using fenestrated-branched stent grafts. J Vasc Surg 2020;72:822-836.e9.

57. Oikonomou K, Kasprzak P, Katsargyris A, et al. Mid-Term Results of Fenestrated/Branched Stent Grafting to Treat Post-dissection Thoraco-abdominal Aneurysms. Eur J Vasc Endovasc Surg 2019;57:102-9.

58. Youssef M, Deglise S, Szopinski P, et al. A Multicenter Experience With a New Fenestrated-Branched Device for Endovascular Repair of Thoracoabdominal Aortic Aneurysms. J Endovasc Ther 2018;25:209-19.

59. Eagleton MJ, Follansbee M, Wolski K, et al. Fenestrated and branched endovascular aneurysm repair outcomes for type II and III thoracoabdominal aortic aneurysms. J Vasc Surg 2016;63:930-42.

60. Verhoeven EL, Katsargyris A, Bekkema F, et al. Editor's Choice - Ten-year Experience with Endovascular Repair of Thoracoabdominal Aortic Aneurysms: Results from 166 Consecutive Patients. Eur J Vasc Endovasc Surg 2015;49:524-31.
61. Dias NV, Sonesson B, Kristmundsson T, et al. Shortterm outcome of spinal cord ischemia after endovascular repair of thoracoabdominal aortic aneurysms. Eur J Vasc Endovasc Surg 2015;49:403-9.

62. Bisdas T, Panuccio G, Sugimoto M, et al. Risk factors for spinal cord ischemia after endovascular repair of thoracoabdominal aortic aneurysms. J Vasc Surg 2015;61:1408-16.

63. Zettervall S, Tenorio ER, Schanzer A, et al. Reinterventions After Branched/Fenestrated Aortic Aneurysm Repairs Are Common and Nondetrimental to Long-term Survival. J Vasc Surg 2021;74:e303-4.

64. Aquina CT, Becerra AZ, Fleming FJ, et al. Variation in outcomes across surgeons meeting the Leapfrog volume standard for complex oncologic surgery. Cancer 2021;127:4059-71.

65. Mirza AK, Tenorio ER, Kärkkäinen JM, et al. Learning curve of fenestrated and branched endovascular aortic repair for pararenal and thoracoabdominal aneurysms. J Vasc Surg 2020;72:423-434.e1.

66. Han Y, Zhang S, Zhang J, et al. Outcomes of Endovascular Abdominal Aortic Aneurysm Repair in Octogenarians: Meta-analysis and Systematic Review. Eur J Vasc Endovasc Surg 2017;54:454-63.

67. Pini R, Gallitto E, Faggioli G, et al. Predictors of perioperative and late survival in octogenarians undergoing elective endovascular abdominal aortic repair. J Vasc Surg 2019;69:1405-11.

68. Makaloski V, Kölbel T, Rohlffs F, et al. Early Outcomes After Branched and Fenestrated Endovascular Aortic Repair in Octogenarians. Eur J Vasc Endovasc Surg 2018;56:818-25.

69. Lagergren E, Chihade D, Zhan H, et al. Outcomes and Durability of Endovascular Aneurysm Repair in Octogenarians. Ann Vasc Surg 2019;54:33-9.

70. Motta F, Oderich GS, Tenorio ER, et al. Fenestratedbranched endovascular aortic repair is a safe and effective option for octogenarians in treating complex aortic aneurysm compared with nonoctogenarians. J Vasc Surg 2021;74:353-362.e1.

71. Richter D, Guasti L, Walker D, et al. Frailty in cardiology: definition, assessment and clinical implications for general cardiology. A consensus document of the Council for Cardiology Practice (CCP), Acute Cardiovascular Care Association (ACCA), Association of Cardiovascular Nursing and Allied Professions (ACNAP), European Association of Preventive Cardiology (EAPC), European Heart Rhythm Association (EHRA), Council on Valvular 
Heart Diseases (VHD), Council on Hypertension (CHT), Council of Cardio-Oncology (CCO), Working Group (WG) Aorta and Peripheral Vascular Diseases, WG e-Cardiology, WG Thrombosis, of the European Society of Cardiology, European Primary Care Cardiology Society (EPCCS). Eur J Prev Cardiol 2021. [Epub ahead of print]. doi: 10.1093/eurjpc/zwaa167.

72. Wang J, Zou Y, Zhao J, et al. The Impact of Frailty on Outcomes of Elderly Patients After Major Vascular Surgery: A Systematic Review and Meta-analysis. Eur J Vasc Endovasc Surg 2018;56:591-602.

73. Ehlert BA, Najafian A, Orion KC, et al. Validation of a modified Frailty Index to predict mortality in vascular surgery patients. J Vasc Surg 2016;63:1595-1601.e2.

74. Thurston B, Pena GN, Howell S, et al. Low total psoas area as scored in the clinic setting independently predicts midterm mortality after endovascular aneurysm repair in male patients. J Vasc Surg 2018;67:460-7.

75. Newton DH, Kim C, Lee N, et al. Sarcopenia predicts poor long-term survival in patients undergoing endovascular aortic aneurysm repair. J Vasc Surg 2018;67:453-9.

76. Kärkkäinen JM, Oderich GS, Tenorio ER, et al. Psoas muscle area and attenuation are highly predictive of complications and mortality after complex endovascular aortic repair. J Vasc Surg 2021;73:1178-1188.e1.

77. Chatterjee S, Shi A, Yoon L, et al. Effect of sarcopenia on survival and spinal cord deficit outcomes after thoracoabdominal aortic aneurysm repair in patients 60 years of age and older. J Thorac Cardiovasc Surg 2021. [Epub ahead of print]. doi: 10.1016/j.jtcvs.2021.05.037.

78. Crawford ES, DeNatale RW. Thoracoabdominal aortic aneurysm: observations regarding the natural course of the disease. J Vasc Surg 1986;3:578-82.

79. Cambria RA, Gloviczki P, Stanson AW, et al. Outcome and expansion rate of 57 thoracoabdominal aortic aneurysms managed nonoperatively. Am J Surg 1995;170:213-7.

80. Hansen PA, Richards JM, Tambyraja AL, et al. Natural history of thoraco-abdominal aneurysm in high-risk patients. Eur J Vasc Endovasc Surg 2010;39:266-70.

81. Kayssi A, DeBord Smith A, Roche-Nagle G, et al. Health-related quality-of-life outcomes after open versus endovascular abdominal aortic aneurysm repair. J Vasc Surg 2015;62:491-8.

82. Coughlin PA, Jackson D, White AD, et al. Meta-analysis of prospective trials determining the short- and midterm effect of elective open and endovascular repair of abdominal aortic aneurysms on quality of life. Br J Surg 2013;100:448-55.

83. Kärkkäinen JM, Sandri GA, Tenorio ER, et al. Prospective assessment of health-related quality of life after endovascular repair of pararenal and thoracoabdominal aortic aneurysms using fenestrated-branched endografts. J Vasc Surg 2019;69:1356-1366.e6.

84. Hiratzka LF, Bakris GL, Beckman JA, et al. 2010 ACCF/AHA/AATS/ACR/ASA/SCA/SCAI/SIR/STS/ SVM guidelines for the diagnosis and management of patients with Thoracic Aortic Disease: a report of the American College of Cardiology Foundation/American Heart Association Task Force on Practice Guidelines, American Association for Thoracic Surgery, American College of Radiology, American Stroke Association, Society of Cardiovascular Anesthesiologists, Society for Cardiovascular Angiography and Interventions, Society of Interventional Radiology, Society of Thoracic Surgeons, and Society for Vascular Medicine. Circulation 2010;121:e266-369.

85. Trans-Atlantic Aortic Research Consortium Investigators. Endovascular repair of intercostal and visceral aortic patch aneurysms following open thoracoabdominal aortic aneurysm repair. J Thorac Cardiovasc Surg 2021. [Epub ahead of print]. doi: 10.1016/j.jtcvs.2021.04.063.

86. Keschenau PR, Kotelis D, Bisschop J, et al. Editor's Choice - Open Thoracic and Thoraco-abdominal Aortic Repair in Patients with Connective Tissue Disease. Eur J Vasc Endovasc Surg 2017;54:588-96.

87. Ribeiro M, Oderich GS, Macedo T, et al. Assessment of aortic wall thrombus predicts outcomes of endovascular repair of complex aortic aneurysms using fenestrated and branched endografts. J Vasc Surg 2017;66:1321-33.

88. Rowse JW, Morrow K, Bena JF, et al. Iliac conduits remain safe in complex endovascular aortic repair. J Vasc Surg 2019;70:424-31.

89. Asciutto G, Aronici M, Resch T, et al. Endoconduits with "Pave and Crack" Technique Avoid Open Ilio-femoral Conduits with Sustainable Mid-term Results. Eur J Vasc Endovasc Surg 2017;54:472-9.

Cite this article as: Tenorio ER, Dias-Neto MF, Lima GBB, Estrera AL, Oderich GS. Endovascular repair for thoracoabdominal aortic aneurysms: current status and future challenges. Ann Cardiothorac Surg 2021;10(6):744-767. doi: 10.21037/acs-2021-taes-24 